\title{
Wheat transcriptome profiling reveals abscisic and gibberellic acid treatments regulate early-stage phytohormone defense signaling, cell wall fortification, and metabolic switches following Fusarium graminearum-challenge
}

\author{
Leann M. Buhrow ${ }^{1}$, Ziying Liư ${ }^{2}$, Dustin Cram¹, Tanya Sharma ${ }^{3}$, Nora A. Foroud ${ }^{4}$, Youlian Pan ${ }^{2 *}$ and
} Michele C. Loewen ${ }^{1,3,5^{*}}$ (D)

\begin{abstract}
Background: Treatment of wheat with the phytohormones abscisic acid (ABA) and gibberellic acid (GA) has been shown to affect Fusarium head blight (FHB) disease severity. However, the molecular mechanisms underlying the elicited phenotypes remain unclear. Toward addressing this gap in our knowledge, global transcriptomic profiling was applied to the FHB-susceptible wheat cultivar 'Fielder' to map the regulatory responses effected upon treatment with $A B A$, an $A B A$ receptor antagonist (AS6), or GA in the presence or absence of Fusarium graminearum (Fg) challenge.

Results: Spike treatments resulted in a total of 30,876 differentially expressed genes (DEGs) identified in 'Fielder' $(26,004)$ and the $F g(4872)$ pathogen. Topology overlap and correlation analyses defined 9689 wheat DEGs as Fgrelated across the treatments. Further enrichment analyses demonstrated that these included expression changes within 'Fielder' defense responses, cell structural metabolism, molecular transport, and membrane/lipid metabolism. Dysregulation of ABA and GA crosstalk arising from repression of 'Fielder' FUS3 was noted. As well, expression of a putative Fg ABA-biosynthetic cytochrome P450 was detected. The co-applied condition of Fg + ABA elicited further upregulation of phytohormone biosynthesis, as well as SA and ET signaling pathways and cell wall/polyphenolic metabolism. In contrast, co-applied Fg + GA mainly suppressed phytohormone biosynthesis and signaling, while modulating primary and secondary metabolism and flowering. Unexpectedly, co-applied Fg + AS6 did not affect ABA biosynthesis or signaling, but rather elicited antagonistic responses tied to stress, phytohormone transport, and FHB disease-related genes.
\end{abstract}

\footnotetext{
* Correspondence: Youlian.Pan@nrc-cnrc.gc.ca; Michele.Loewen@nrc.ca

${ }^{2}$ National Research Council of Canada, Digital Technologies Research Centre,

1200 Montreal Road, Ottawa, ON K1A OR6, Canada

'National Research Council of Canada, Aquatic and Crop Resources

Development Research Centre, 110 Gymnasium Place, Saskatoon, SK S7N

OM8, Canada

Full list of author information is available at the end of the article
}

C C The Author(s). 2021 Open Access This article is licensed under a Creative Commons Attribution 4.0 International License, which permits use, sharing, adaptation, distribution and reproduction in any medium or format, as long as you give appropriate credit to the original author(s) and the source, provide a link to the Creative Commons licence, and indicate if changes were made. The images or other third party material in this article are included in the article's Creative Commons licence, unless indicated otherwise in a credit line to the material. If material is not included in the article's Creative Commons licence and your intended use is not permitted by statutory regulation or exceeds the permitted use, you will need to obtain permission directly from the copyright holder. To view a copy of this licence, visit http://creativecommons.org/licenses/by/4.0/ The Creative Commons Public Domain Dedication waiver (http://creativecommons.org/publicdomain/zero/1.0/) applies to the data made available in this article, unless otherwise stated in a credit line to the data. 
Conclusions: Observed exacerbation (misregulation) of classical defense mechanisms and cell wall fortifications upon ABA treatment are consistent with its ability to promote FHB severity and its proposed role as a fungal effector. In contrast, GA was found to modulate primary and secondary metabolism, suggesting a general metabolic shift underlying its reduction in FHB severity. While AS6 did not antagonize traditional ABA pathways, its impact on host defense and Fg responses imply potential for future investigation. Overall, by comparing these findings to those previously reported for four additional plant genotypes, an additive model of the wheat-Fg interaction is proposed in the context of phytohormone responses.

Keywords: Wheat, Triticum aestivum, Fusarium graminearum, Fusarium head blight, Phytohormone, Abscisic acid, Gibberellic acid, Differentially expressed genes, RNA-seq

\section{Background}

Fusarium head blight (FHB), one of the more prevalent diseases of wheat (Triticum aestivum L.), is the result of infection of wheat heads by the hemi-biotrophic ascomycetous Fusarium graminearum $(F g)$ and related species [1]. FHB remains a significant pathogenic threat to the agricultural industry; $F g$ infection decreases grain yields and deposits mycotoxins [2, 3]. Wheat breeding programs aimed at addressing FHB have resulted in incremental benefits to date [4], while chemical control measures remain limited by host-developmental requirements of the pathogen [5]. Thus, it is imperative that we expand our understanding of these host-pathogen interactions to identify new sources of resistance and antifungal molecular targets.

The transcriptomic responses of FHB-susceptible and -resistant wheat varieties in response to $F g$ challenge have been investigated extensively over the course of the last decade (reviewed in [6]; and more recently [7-11]). Together these studies highlight a breadth of host-responses that include modulation of primary metabolism and photosynthesis, transcriptional and translational regulators, traditional plant defense responses (both phytohormone-associated and pathogenesis-related protein targets), and detoxification genes. The role of the classical defense phytohormones, salicylic acid (SA), jasmonic acid (JA), and ethylene (ET) in the wheat defense response to $F g$-challenge has been extensively described with a consensus model of early biotrophic SA followed by later stage necrotrophic JA/ET responses $[6,8$, 9]. Nonetheless, the role of ET remains in question, with conflicting reports highlighting mediation of both resistance [3] and susceptibility [12] in early and late responses [9].

In agreement with independent research groups investigating other wheat varieties and FHB disease stages [9, 13, 14], our previous report described drastic alterations of phytohormone profiles in the FHB-susceptible $T$. aestivum cultivar 'Fielder' when challenged with $\mathrm{Fg}$ [15]. Two such FHB-regulated phytohormones, when coapplied with pathogen challenge, modulated disease severity and spread where abscisic acid (ABA) promoted infection and gibberellic acid (GA) reduced infection $[14,15]$. It has been established that Fusarium spp. can themselves produce ABA [14], GA [16], auxin (indole acetic acid; IAA [17]), and cytokinins (CK [18];), while also encoding both 1-aminocyclopropane carboxylic acid (ACC) synthases and deaminases potentially involved in ET biosynthesis [19]. Therefore, although phytohormones may be traditionally thought to serve as plant host signaling and defense molecules, Fusarium spp. may hijack or dysregulate phytohormone metabolism to establish or promote infection.

Whether exogenously applied, or derived from wheat or Fusarium, the mechanisms by which ABA and GA alter host resistance and susceptibility during $F g$ infection remains unclear. As ABA and GA antagonistically regulate many plant developmental processes and elicit opposing changes to 'Fielder' FHB disease, it is tempting to hypothesize that these phytohormones may dysregulate each other's biosynthesis, signaling, and crosstalk with classical defense phytohormones. In the present study, this hypothesis is largely discounted as robust phytohormone-related gene expression antagonism is not observed. Instead, more than 30,000 DEGs are identified across both wheat and $F g$ genomes in response to the seven combinatorial treatment types. To deconvolute this complex data set, differential expression feature extraction (DEFE) was employed, ultimately allowing DEGs highly enriched upon $\mathrm{Fg}$ challenge to be identified and further analyzed for association networks across treatments. Subsequently, the DEGs elicited by $F g$ challenge, ABA or GA treatment alone, and the combined effects of both the pathogen and phytohormone were individually targeted. This analysis also considers DEGs arising upon co-application of the antagonist of ABA receptors, AS6 [20]. Ultimately, transcriptomic responses of five wheat genotypes upon Fusarium challenge are compared to further contribute to a consensus model of this plant-pathogen interactions. We discuss how transcriptomic changes elicited by ABA and GA impact such a consensus model and may contribute to the modulation of FHB symptoms previously reported in Qi et al. [14] and Buhrow et al. [15].

\section{Results}

\section{Transcriptome overview}

FHB-susceptible wheat cultivar 'Fielder' spikes were treated with ABA (condition: ABA), GA (condition: GA), or $F g$ (condition: $F g$ ) alone. Additionally, 'Fielder' 
spikes were treated with a combination of $F g$ and $A B A$ (condition: $F g$ + ABA), GA (condition: $F g+\mathrm{GA}$ ) or an ABA receptor antagonist (condition: $F g$ + AS6). RNAseq reads (Additional file $1 \mathrm{Tab}$ 'S1') were remapped to a combination of the wheat genome (IWGSC RefSeq v1.0 [21];) and Fusarium graminearum (str. PH-1) resulting in $97 \%$ of the reads being successfully mapped to the two reference genomes (Additional file 1, Tab 'S1'). Fgchallenge and phytohormone application resulted in consistent and distinguishable changes to the 'Fielder' transcriptome (Fig. 1). After normalization of the read counts, a total of 30,876 differentially expressed genes (DEGs) were identified based on the criteria specified in the Methods section, including 4872 from F. graminearum and 26,004 from wheat (Table 1; Additional files $2 \& 3$ ).

Toward assessing the relative effects of the different treatments, differential expression feature extraction (DEFE [22];) analysis was performed. Among the $729\left(3^{6}\right)$ theoretically possible ' $\mathrm{M}$ ' DEFE-patterns for the six treatments as they compared with mock water treatment (MT), 266 had one or more genes. A list of 30 highly populated DEFE patterns collectively contain 17,170 (66\%) of the wheat DEGs and 4839 (99\%) of the $F g$ DEGs (Table 2). The frequency distribution in other DEFE patterns is available in Additional files 2 \& 3 Tab 'DEFE_stats'. Observations of the patterns containing the highest number of wheat DEGs included those up- or down-regulated by the pathogen irrespective of one or more coapplied compounds [M001100 (1736 DEGs), M202222 (1641 DEGs), M001111 (1593 DEGs), M002222 (1181 DEGs)]. At a high level, the DEFE and principal component analyses (PCA discussed in next section; Fig. 1b) supported the fact that a high number of DEGs were derived from pathogen challenge, while fewer DEGs were derived from ABA or GA treatment. Similarly, $F g$ DEGs were highly enriched with four DEFE patterns, which collectively accounted for $97 \%$ of $F g$ DEGs (Table 1, Additional file 3).

\section{Wheat DEGs highly correlated with $\mathrm{Fg}$ challenge}

Wheat transcriptomic responses were most clearly divided based on the presence or absence of $F g$ challenge, contributing $57.2 \%$ of the variance in the PCA (Fig. 1b). Through topology overlap analysis using WGCNA [23], 58 DEG clusters were identified in this dataset with 17 being significantly correlated $(p<5 \times$ $\left.10^{-2} ; 12,138 \mathrm{DEGs}\right)$ to $\mathrm{Fg}$ treatment (Additional file 2 Tab 'WheatDEGs' @Col-M,N \& Tab 'moduleTraitCor'). This finding was further validated by correlation analysis which revealed 10,609 individual $F g$ correlated DEGs. Of these, 9689 (91\%) were common to the $\mathrm{Fg}$ correlated topology overlap clusters and were thus considered ' $F g$-related genes' (Additional file 2 Tab 'WheatDEGs' @Col-O). The majority
(83\%) of these $9689 \mathrm{Fg}$-related 'Fielder' genes fall into the most informative ' $M$ ' DEFE patterns listed in Table 2.

To further understand the relationship of DEGs identified by topology overlap, gene association network analysis was performed using the WGCNA R package [23]. The top $1 \%$ of the topology overlap matrix was considered in the entire network consisting of 10,373 wheat DEGs, connected by $3,370,764$ edges. Of these, 8349 (80\%) were $F g$-related genes. The top 2621 genes having connection degrees of 1000 or higher were considered to be potential key regulators of the associated group of genes, and are referred to as key hub genes (highlighted in Additional file 2 Tab 'WheatDEGs'@Col-P). An enrichment index analysis of gene groups was performed on the network genes as described in Pan et al., [8]. Among the highly enriched group of genes in the network were alkaline shock protein 23 , clathrin assembly family protein, D-glycerate dehydrogenase / hydroxypyruvate reductase, photosystem II $22 \mathrm{kDa}$, chloroplastic, PISTILLATA-like MADS-box transcription factor, fatty acid hydroxylase, fimbrin-like protein 2, pollen allergen, glyoxal oxidase, jasmonate ZIM domain proteins, $\mathrm{C} 2$ domain-containing protein, yellow stripe-like transporters, and pectinesterase (Additional file 4). The B3 domain-containing transcription factor FUS3 gene (TraesCS3D01G249100) was among the key hub genes, with 2281 immediate neighborhood genes in the network. It was significantly downregulated by all conditions that include $F g$-treatment (adj $p<2 \times 10^{-14}$ ) (Additional file 5: Figure S1). Interestingly, all conditions that included $F g$-treatment also expressed a putative fungal ABA biosynthetic cytochrome P450 homolog (FGRAMPH1-01 T26277, Additional file 5: Figure S2B).

\section{$A B A$ and GA treatments alone elicited diverse metabolic changes to the wheat transcriptome}

To characterize the impacts of ABA and GA application alone, these data were compared to MT. Neither phytohormone treatment elicited wheat responses as strong as Fg challenge (Fig. 1a \& b; Table 1); however, ABA downregulated nearly twice as many wheat genes as it upregulated, while GA modified approximately equal numbers of genes up and down (Table 1, Fig. 1c \& d), consistent with the hormone-responsive trends recently reported on the FHB-susceptible wheat cultivar 'Roblin' [11]. ABA treatment most notably led to down-regulation of 3079 (43\%) wheat DEGs that were down-regulated by $\mathrm{Fg}$ as well as up-regulation of 1457 (21\%) wheat DEGs that were up-regulated by $F g$ (Fig. 2). Furthermore, ABA elicited few opposing effects when compared to the $F g$ condition (GO enrichment of these DEGs found in 

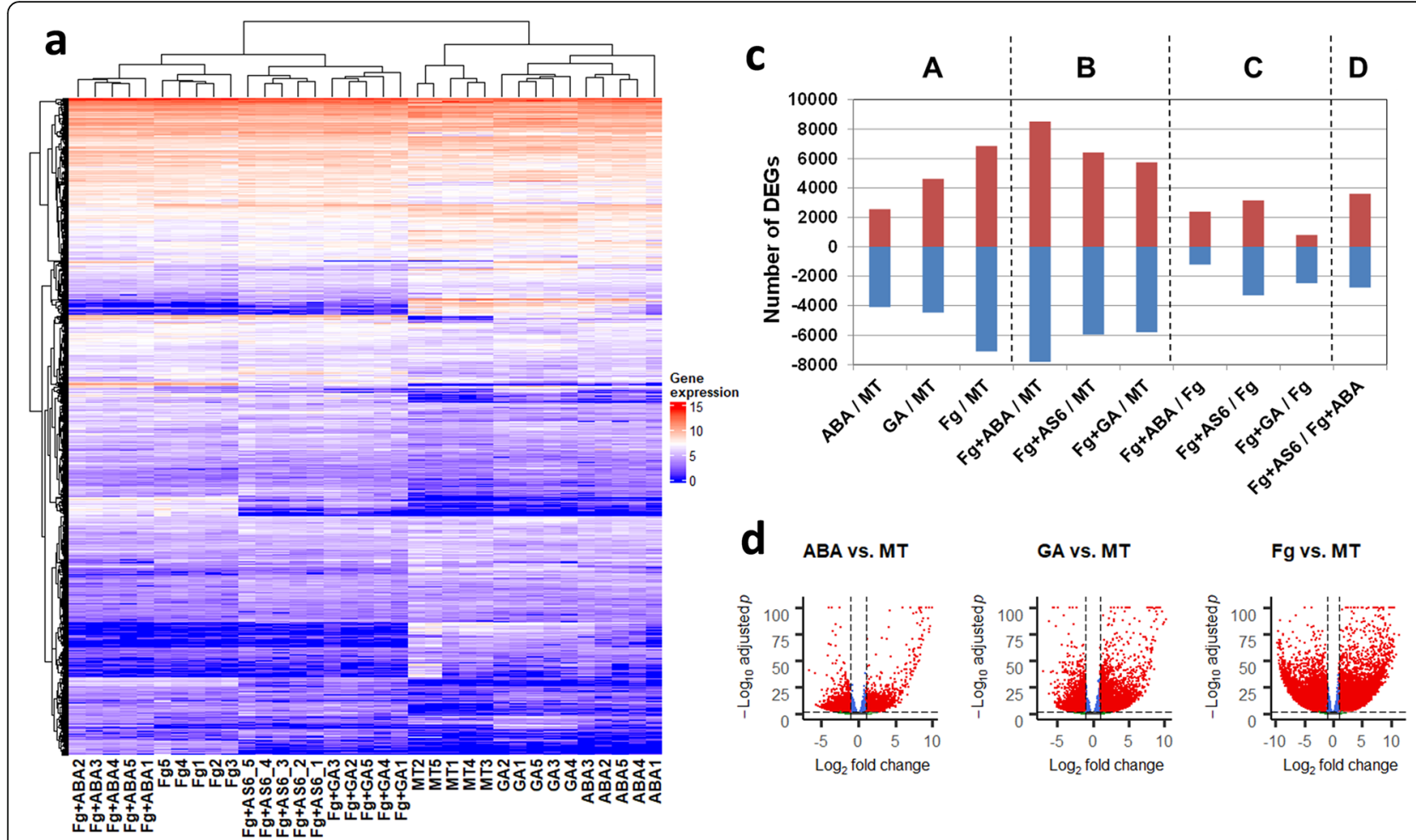

Fg+ABA vs. MT

Fg+AS6 vs. MT

Fg+GA vs. MT

\section{b}
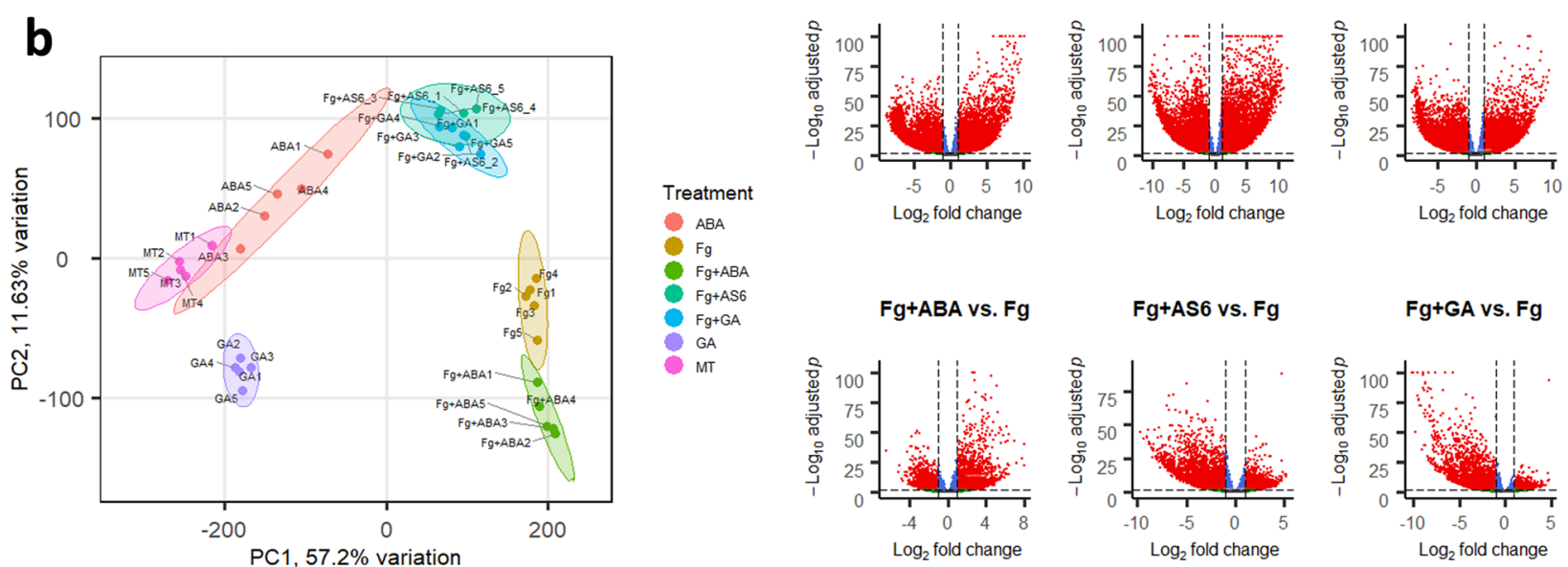

Fg+ABA vs. Fg
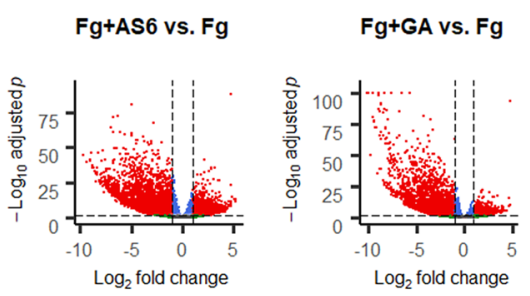

Fig. 1 Transcriptome overview. Gene expression levels were $\log _{2}$ transformed read counts. a heatmap of wheat DEGs; $\mathbf{b}$ PCA of wheat DEGs; $\mathbf{c}$ wheat DEG distribution over the 10 pairwise comparisons, A: "alone" conditions compared with MT, B: co-applications compared with MT, C: co-applications compared with Fg alone, D: comparison between two co-applications; $\mathbf{d}$ volcano plots of wheat DEGs of the first nine pairwise comparisons (sub-panel A, B, C of panel d), adjusted $p$ values less than $10^{-100}$ were displayed as $10^{-100}$

Additional file 6 Tab 'FU2'). GA treatment elicited 20$25 \%$ of wheat DEGs in the same directionality as the $\mathrm{Fg}$ condition, particularly down-regulated 204 (3\%) DEGs that were up-regulated by $F g$ and conversely up-regulating 286 (4\%) DEGs that were down-regulated by Fg (Fig. 2; GO enrichment of these DEGs found in Additional file 6 Tab 'FU4').
Fg challenge with $\mathrm{ABA}$ and GA co-application respectively enhanced and suppressed wheat DEGs elicited by $\mathrm{Fg}$ alone The wheat transcriptomic changes elicited by ABA $(F g+$ $\mathrm{ABA})$ and $\mathrm{GA}(\mathrm{Fg}+\mathrm{GA})$ co-application in the presence of $\mathrm{Fg}$ pathogen challenge were collectively analyzed as compared to MT. This enabled a direct comparison of gene regulation events mediated by phytohormones 
Table 1 Number of DEGs arising from each pairwise comparison. For each treatment condition and appropriate reference background, mock-treated (MT) or Fg challenged (Fg), the total number of DEGs are presented. DEGs are further subdivided based on mapping to the wheat or Fg genome and directionality of the expression changes (up-regulated or down-regulated)

\begin{tabular}{|c|c|c|c|c|c|c|c|c|c|c|c|}
\hline \multirow[b]{2}{*}{$\begin{array}{l}\text { DEG } \\
\text { Population }\end{array}$} & \multirow[b]{2}{*}{ Regulation } & \multicolumn{10}{|c|}{ Condition } \\
\hline & & $\begin{array}{l}\text { ABA/ } \\
\text { MT }\end{array}$ & $\begin{array}{l}\mathrm{GA} / \\
\mathrm{MT}\end{array}$ & $\begin{array}{l}\mathrm{Fg} / \\
\text { MT }\end{array}$ & $\begin{array}{l}\mathrm{Fg}+\mathrm{ABA} / \\
\mathrm{MT}\end{array}$ & $\begin{array}{l}\mathrm{Fg}+\mathrm{AS6/} \\
\mathrm{MT}\end{array}$ & $\begin{array}{l}\mathrm{Fg}+\mathrm{GA} / \\
\mathrm{MT}\end{array}$ & $\begin{array}{l}\mathrm{Fg}+\mathrm{ABA} / \\
\mathrm{Fg}\end{array}$ & $\begin{array}{l}\mathrm{Fg}+\mathrm{GAl} \\
\mathrm{Fg}\end{array}$ & $\begin{array}{l}\mathrm{Fg}+\mathrm{AS6/} \\
\mathrm{Fg}\end{array}$ & $\begin{array}{l}F g+A S 6 / F g+ \\
A B A\end{array}$ \\
\hline \multirow[t]{2}{*}{ Total } & Up & 2566 & 4623 & 8691 & 11772 & 7668 & 10560 & 2477 & 1703 & 3163 & 3602 \\
\hline & Down & 4098 & 4473 & 7100 & 7810 & 5962 & 5798 & 1213 & 2472 & 3325 & 3330 \\
\hline \multirow[t]{2}{*}{ Wheat } & Up & 2566 & 4623 & 6855 & 8529 & 6410 & 5743 & 2398 & 806 & 3146 & 3602 \\
\hline & Down & 4098 & 4473 & 7100 & 7810 & 5962 & 5798 & 1213 & 2471 & 3299 & 2773 \\
\hline \multirow[t]{2}{*}{$\mathrm{Fg}$} & Up & 0 & 0 & 1836 & 3243 & 1258 & 4817 & 79 & 897 & 17 & 0 \\
\hline & Down & 0 & 0 & 0 & 0 & 0 & 0 & 0 & 1 & 26 & 557 \\
\hline
\end{tabular}

Table 2 Number of genes in highly informative Differential Expression Feature Extraction (DEFE) patterns M (ABA/MT, GA/MT, Fg/MT, $F g+$ ABA/MT, Fg + AS6/MT, Fg + GA/MT)

\begin{tabular}{|c|c|c|c|c|}
\hline Pattern & Wheat genes & FHB related wheat genes ${ }^{a}$ & $\mathrm{Fg}$ genes & Description \\
\hline M000001 & 289 & $41 \%$ & 1589 & up-regulated by $F g+G A$, but not by them separately \\
\hline M000002 & 111 & $31 \%$ & 0 & down-regulated by $F g+G A$, but not by them separately \\
\hline M000010 & 994 & $28 \%$ & 6 & up-regulated by $F g+A S 6$, but not by them separately \\
\hline M000020 & 248 & $37 \%$ & 0 & down-regulated by $F g+A S 6$, but not by them separately \\
\hline M000100 & 698 & $19 \%$ & 40 & up-regulated by $F g+A B A$, but not by them separately \\
\hline M000200 & 960 & $21 \%$ & 0 & down-regulated by $F g+A B A$, but not by them separately \\
\hline M001000 & 378 & $44 \%$ & 5 & up-regulated by $\mathrm{Fg}$ alone \\
\hline M002000 & 429 & $39 \%$ & 0 & down-regulated by $F g$ alone \\
\hline M010000 & 1306 & $4 \%$ & 0 & up-regulated by GA alone \\
\hline M020000 & 1163 & $3 \%$ & 0 & down-regulated by GA alone \\
\hline M100000 & 254 & $7 \%$ & 0 & up-regulated by $A B A$ alone \\
\hline M200000 & 218 & $3 \%$ & 0 & down-regulated by $A B A$ alone \\
\hline M001111 & 1593 & $97 \%$ & 1165 & up-regulated by $F g$, with or without a hormone \\
\hline M002222 & 1181 & $94 \%$ & 0 & down-regulated by $F g$, with or without a hormone \\
\hline M000111 & 309 & $77 \%$ & 72 & up-regulated by $\mathrm{Fg}$ in combination of any of the three hormones \\
\hline M000222 & 29 & $55 \%$ & 0 & down-regulated by $\mathrm{Fg}$ in combination of any of the three hormones \\
\hline M010001 & 10 & $0 \%$ & 0 & up-regulated by $\mathrm{GA}$, with or without $\mathrm{Fg}$ \\
\hline M020002 & 10 & $0 \%$ & 0 & down-regulated by GA, with or without $F g$ \\
\hline M100100 & 9 & $0 \%$ & 0 & up-regulated by $A B A$, with or without $F g$ \\
\hline M200200 & 22 & $0 \%$ & 0 & down-regulated by $A B A$, with or without $F g$ \\
\hline M111111 & 750 & $42 \%$ & 0 & up-regulated by all treatments \\
\hline M222222 & 964 & $42 \%$ & 0 & down-regulated by all treatments \\
\hline M001100 & 1736 & $32 \%$ & 0 & up-regulated by Fg, but GA or AS6 neutralized/suppressed the effect of Fg \\
\hline M002200 & 543 & $38 \%$ & 0 & down-regulated by $F g$, but GA and AS6 neutralized/suppressed the effect of $F g$ \\
\hline M000101 & 45 & $58 \%$ & 1315 & up-regulated by $\mathrm{Fg}$ in combination of either $\mathrm{ABA}$ or $\mathrm{GA}$ \\
\hline M000202 & 27 & $63 \%$ & 0 & down-regulated by $\mathrm{Fg}$ in combination of either $\mathrm{ABA}$ or GA \\
\hline M001101 & 552 & $81 \%$ & 647 & up-regulated by $F g$ with or without combination of either ABA or GA \\
\hline M002202 & 323 & $73 \%$ & 0 & down-regulated by $\mathrm{Fg}$ with or without combination of either ABA or GA \\
\hline M101111 & 378 & $70 \%$ & 0 & up-regulated by all treatment excepting GA alone \\
\hline M202222 & 1641 & $86 \%$ & 0 & down-regulated by all treatment excepting GA alone \\
\hline
\end{tabular}

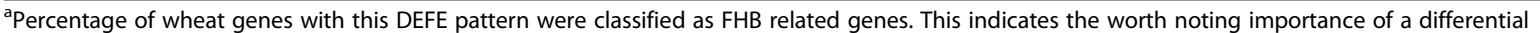
expression profile 
a

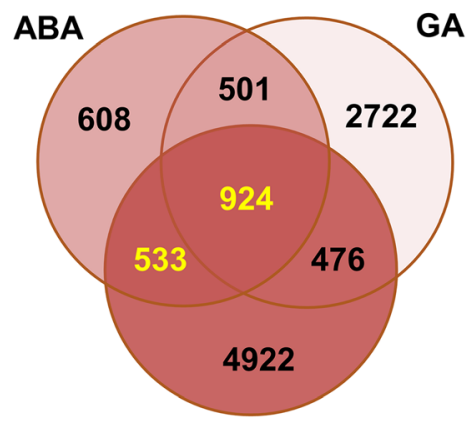

$F g$
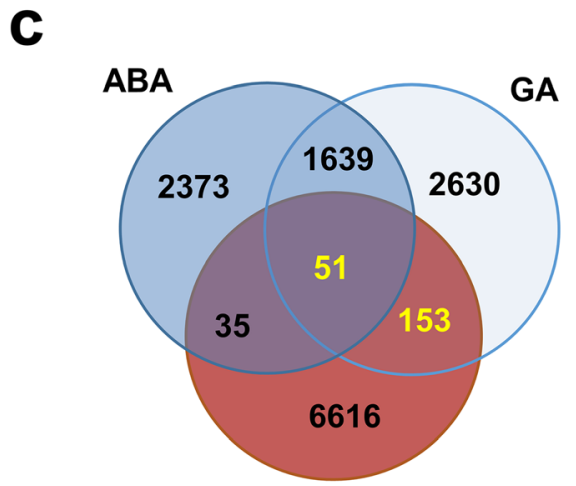

$F g$ b

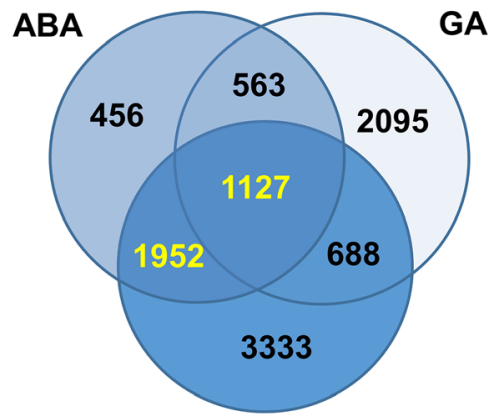

$F g$
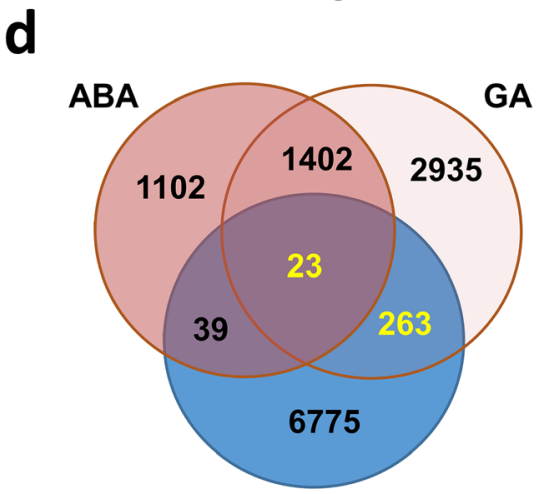

$F g$

Fig. 2 Share of Fg alone up- or down- regulated genes that are up- and down- regulated under ABA and GA alone conditions, with all DEGs calculated compared to mock treated (MT). In all cases, up-regulated genes are highlighted in shades of red, down-regulated genes are highlighted in shades of blue. DEGs called in text are yellow. a Up-regulated DEGS. b Down-regulated DEGS. Opposing DEGs: $\mathbf{c}$ up-regulated by Fg but down-regulated by ABA and GA; d down-regulated by $\mathrm{Fg}$ but up-regulated by $\mathrm{ABA}$ and $\mathrm{GA}$

alone to those arising from the condition where all three components of the 'plant-pathogen-phytohormone' interaction were present together. In the case of responses common to the $F g, F g+\mathrm{ABA}$ and $F g+\mathrm{GA}$ treatments $(F g \cap[F g+\mathrm{ABA}] \cap[F g+\mathrm{GA}]), F g+\mathrm{ABA}$ and $F g+\mathrm{GA}$ jointly up-regulated $3751(55 \%)$ wheat DEGs that were up-regulated by $\mathrm{Fg}$ alone and down-regulated 4696 (66\%) DEGs that were down-regulated by $F g$ alone (highlighted in Additional file 5: Figure S3A \& B). The co-applications also elicited opposing wheat DEGs; $F g$ + ABA up-regulated 63 and down-regulate 4. DEGs, and Fg + GA up-regulated 2 and downregulated 5 DEGs that were down-regulated and upregulated by $\mathrm{Fg}$ alone (highlighted in Additional file 5: Figure S3C \& D). These changes, along with nonoverlapping DEGs, manifested in an increase upon $F g+\mathrm{ABA}$ and slight reduction upon $\mathrm{Fg}+\mathrm{GA}$ treatments in the overall DEG counts when compared to $\mathrm{Fg}$ alone (Fig. 1c; Table 1). When further considering DEGs elicited by the phytohormone, pathogen, and co-applied conditions, the overlap in elicited wheat transcriptomic changes was over 90\% DEGs common in the case of $\mathrm{ABA}(\mathrm{ABA} \cap F g \cap[F g+\mathrm{ABA}])$ and over

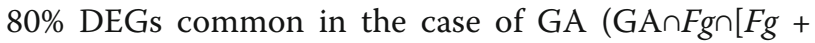
GA]; Additional file 5: Figure S4).

A second DEFE analysis ('F' DEFE-patterns)' was carried out as compared to $\mathrm{Fg}$ challenge to describe the additive DEGs elicited upon phytohormone co-application (Table 3; Additional file 2 Tab 'DEFE_stat'). The $F g+$ ABA co-application significantly enhanced 316 upregulated and 572 down-regulated $F g$-elicited wheat DEGs (Fig. 3a \& d) while also suppressing 142 up-regulated and 279 down-regulated $F g$-elicited wheat DEGs (Fig. 3c \& b). The $F g+\mathrm{GA}$ co-application significantly enhanced 152 up-regulated and 349 down-regulated $F g$-elicited wheat DEGs (highlighted in Fig. 4a \& d) while also suppressing 2232 (33\%) up-regulated and 7 down-regulated Fg-elicited wheat DEGs (highlighted in Fig. 4c \& b). These findings clearly demonstrate the additive effects of $F g+\mathrm{ABA}$ and the decremental effects of $F g+\mathrm{GA}$ on the 'Fielder' transcriptome challenged with $\mathrm{Fg}$ consistent with the observations that ABA co-application predominantly enhances, and GA predominantly represses, the wheat transcriptome (Fig. 1d). 
Table 3 Number of genes with highly informative DEFE patterns on enhancement or suppression of a hormone in co-application with $F g$ : $F(F g+$ ABA_vs_Fg, Fg + AS6_vs_Fg, Fg + GA_vs_Fg)

\begin{tabular}{|c|c|c|c|}
\hline Pattern & Wheat & $\mathrm{Fg}$ & Description \\
\hline F001 & 212 & 856 & Enhanced by GA \\
\hline F002 & 300 & 1 & Suppressed by GA \\
\hline F010 & 2046 & 6 & Enhanced by AS6 \\
\hline F020 & 1036 & 24 & Suppressed by AS6 \\
\hline F100 & 1639 & 39 & Enhanced by ABA \\
\hline F200 & 1038 & 0 & Suppressed by ABA \\
\hline F011 & 381 & 2 & Enhanced by both AS6 and GA, but not by ABA \\
\hline F022 & 2134 & 0 & Suppressed by both AS6 and GA, but not by ABA \\
\hline F101 & 63 & 31 & Enhanced by both ABA and GA, but not by AS6 \\
\hline F202 & 14 & 0 & Suppressed by both ABA and GA, but not by AS6 \\
\hline F110 & 548 & 3 & Enhanced by both $A B A$ and $A S 6$, but not GA \\
\hline F220 & 93 & 0 & Suppressed by both ABA and AS6, but not by GA \\
\hline F111 & 123 & 6 & Enhanced by all three hormones \\
\hline F222 & 13 & 0 & Suppressed by all three hormones \\
\hline F012 & 1 & 0 & Enhanced by AS6, but suppressed by GA \\
\hline F021 & 1 & 2 & Suppressed by AS6, but enhanced by GA \\
\hline F102 & 3 & 0 & Enhanced by ABA, but suppressed by GA \\
\hline F201 & 8 & 0 & Suppressed by both ABA, but enhanced by GA \\
\hline F120 & 16 & 0 & Enhanced by ABA, but suppressed by AS6 \\
\hline F210 & 29 & 0 & Suppressed by both ABA, but enhanced by AS6 \\
\hline F122 & 6 & 0 & Enhanced by ABA, but suppressed by both AS6 and GA \\
\hline F211 & 18 & 0 & Suppressed by ABA, but enhanced by both AS6 and GA \\
\hline
\end{tabular}

Application of $A B A$ and GA elicited wheat DEGs mapped to chromosome $6 \mathrm{BL}$ consistent with their modulation of later-stage FHB phenotype

To understand if the observed modulation of FHB spread by ABA and GA might be tied to a characterized FHB-resistance quantitative trait locus (QTL), DEGs from all treatments were mapped and binned based on chromosomal arm (Fig. 5a \& b). Density as a function of physical length [21] was relatively monodisperse with $26.1 \pm 6.4$ (average \pm one standard deviation) upregulated DEGs and 25.9 \pm 6.3 down-regulated DEGs calculated per $\mathrm{Mb}$, excluding Chr5AS for which fewer induced DEGs were mapped (Additional file 5: Figure S5). DEGs appear to map uniformly between the A, B, and $\mathrm{D}$ genomes with the exceptions of up-regulated DEGs of Chr 4AL, 5AS, 6BL, and 7BS and downregulated DEGs of Chr $4 \mathrm{AL}$ and Chr 7BS compared to their homoeologs (Fig. 5a \& b). Chr 6BL exhibited differential trends with an over-representation of induced $\mathrm{DEGs}$ in $\mathrm{ABA}$ and $\mathrm{ABA} \cap F g \cap[F g+\mathrm{ABA}]$ treatment groups, while also having an over-representation of down-regulated DEGs upon $F g+$ GA treatment. As these early-stage results are in agreement with the late-stage
FHB phenotypes elicited by these treatments, it is possible that the genetic underpinnings may be partially attributed to genes located on Chr 6BL. In wheat and barley, this chromosome arm encodes ABA and GA opposite-regulated $\alpha$-amylase genes [24], an ABA 8 '-hydroxylase important for ABA catabolism and seed germination [25], and ABA responsive yet-uncharacterized gene(s) that are involved in dormancy [26] (Fig. 6).

\section{Application of ABA receptor antagonist, AS6, elicits few Fg DEGs but recapitulates the wheat DEGs induced by $A B A$ and reduced by $G A$ co-application}

A synthetic ABA receptor antagonist would potentially serve as an excellent tool compound; however, AS6, an effective ABA receptor inhibitor in Arabidopsis [20], has not been established as a functional antagonist in wheat. $F g+$ AS6 enhanced the expression of 604 (8.8\%) up-regulated and 904 (13\%) down-regulated wheat DEGs elicited by $F g$ alone (Additional file 5: Figure S6A \& D), while also suppressing 2685 (39\%) up-regulated and 47 (0.66\%) downregulated wheat DEGs elicited by $F g$ alone (Additional file 5: Figures S6C \& B). However, this treatment elicited few antagonistic DEGs with only 47 (1.5\%) up- 


\section{a}

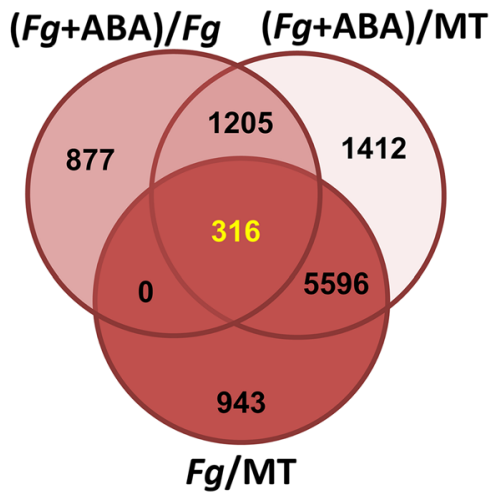

C

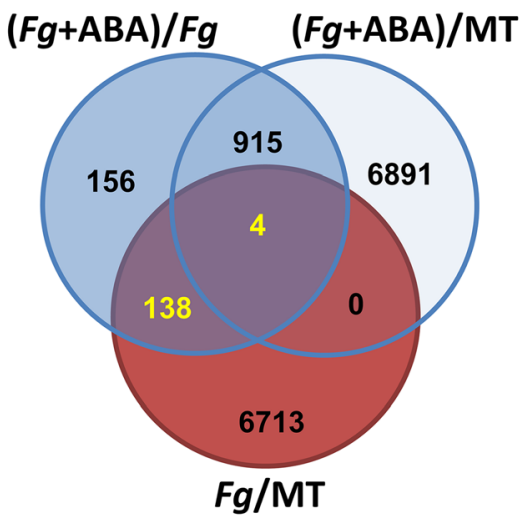

b

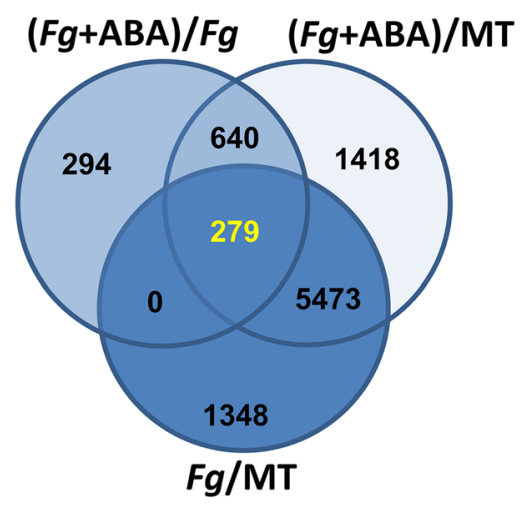

C

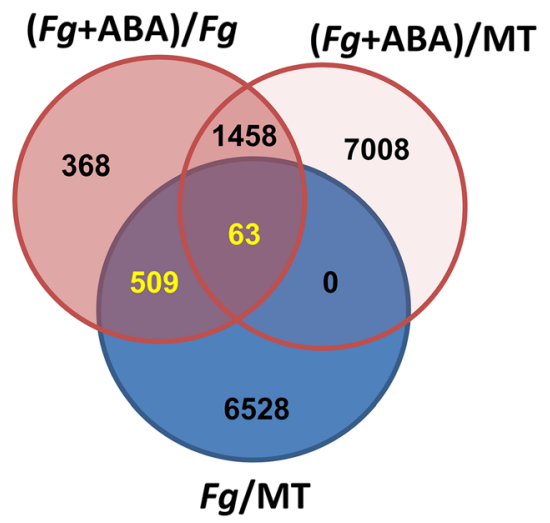

Fig. 3 Comparison of gene regulation elicited by ABA in the presence of Fg, with DEGs calculated compared to mock treated (MT) as well as to Fg. In all cases, up-regulated genes are highlighted in shades of red, and down-regulated genes are highlighted in shades of blue. DEGs called in text are yellow. a Distribution of all up-regulated genes by $\mathrm{Fg}+\mathrm{ABA}$ as compared to $\mathrm{Fg}$, or $\mathrm{Fg}+\mathrm{ABA}$ and $\mathrm{Fg}$-alone compared to MT. b Distribution of all down-regulated genes by $\mathrm{Fg}+\mathrm{ABA}$ as compared to $F g$, or Fg + ABA and $F g$-alone compared to MT. c Distribution of up-regulated genes by $F g$-alone, but down-regulated by $F g+A B A$ as they compared to $\mathrm{Fg}$ or to MT. d Distribution of all down-regulated genes by $\mathrm{Fg}$-alone, but up-regulated by $\mathrm{Fg}+\mathrm{ABA}$ as they compared to $\mathrm{Fg}$ or to MT.

regulated and $22(0.67 \%)$ down-regulated, when compared to those elicited by $F g+\mathrm{ABA}$ (Additional file 5: Figure S6C and D; commonly elicited DEGs by these treatments may also be found in Additional file 5: Figure S6A and B and Figure S7). At this global transcriptomic level, evidence of AS6 antagonism on wheat ABA receptors was not clearly observed; therefore, comparisons of DEGs (Additional file 5: Figure S7) and DEFE patterns (Table 2) elicited by co-applied $\mathrm{Fg}+\mathrm{AS} 6$ and $\mathrm{Fg}+\mathrm{ABA}$, compared to $F g$ alone, were conducted. Of the 316 DEGs upregulated by $F g$ alone and further enhanced by $F g+\mathrm{ABA}$, 11 DEGs were suppressed by $F g$ + AS6 (highlighted in Additional file 5: Figure S6C). These included a $\gamma$ glutamyl phosphate reductase (TraesCS3B01G395900) and a eukaryotic peptide chain release factor subunit 1-1 (TraesCS1A01G235000; both genes adjusted $p<10^{-21}$, Additional file 5: Figure S9) found in the DEFE patterns M111100 and M111101 (Additional file 5; Figure S8). Conversely, among the 279 DEGs down-regulated by $F g$ and further repressed by $F g+\mathrm{ABA}, 29 \mathrm{DEGs}$ were enhanced by $\mathrm{Fg}+$ AS6 (Additional file 5: Figure S6D). These included four DEGs that were repressed by $A B A$ in the presence or absence of $F g$ challenge ( $F g+\mathrm{ABA}$ or ABA treatments) as evidenced by DEFE pattern M202202 and M222202 (Additional file 5: Figure S10). They include a BDX gene (TraesCS7B01G353600), transmembrane protein (TraesCS7A01G453100), leucine-rich repeat receptor-like protein kinase family protein (TraesCS3D01G040100), and nitrate transporter 1.1 (TraesCS1B01G225000; expression highly significantly different from $\mathrm{Fg}+\mathrm{ABA}$ with adjusted $p<$ $10^{-11}$, Additional file 5: Figure $S 9$ ). Furthermore, with respect to the $F g$ pathogen genes (Additional file 5: Figure S2A), $F g+$ AS6 repressed 26 DEGs including 11 putative oxidoreductases $\left(p<10^{-4}\right.$, Additional file 6 Tab 'F1', Additional file 7) and enhanced 17 DEGs including three putative O-glycosyl hydrolases $\left(p<4 \times 10^{-3}\right.$, Additional file $6 \mathrm{Tab}$ ' $\mathrm{F} 2$ '). This treatment also repressed 557 DEGs up-regulated by $F g+$ $\mathrm{ABA}$ and elicited no $F g+\mathrm{ABA}$ - opposing effect (Table 1). 


\section{a}

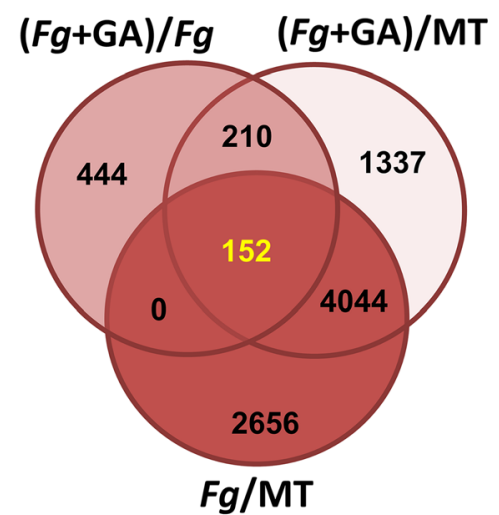

C

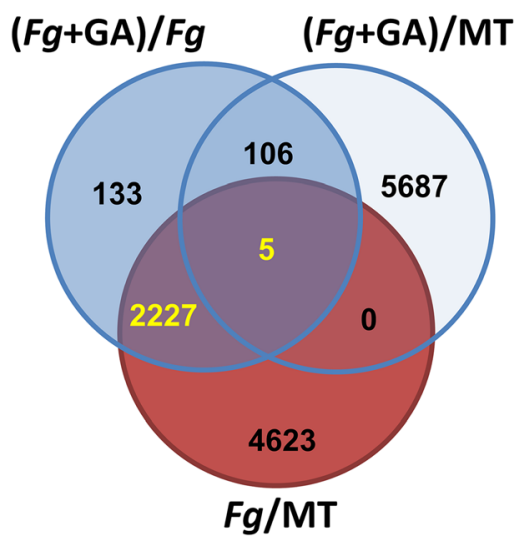

\section{b}

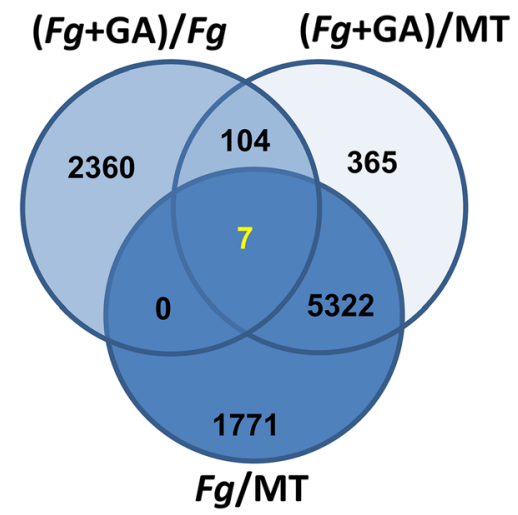

d

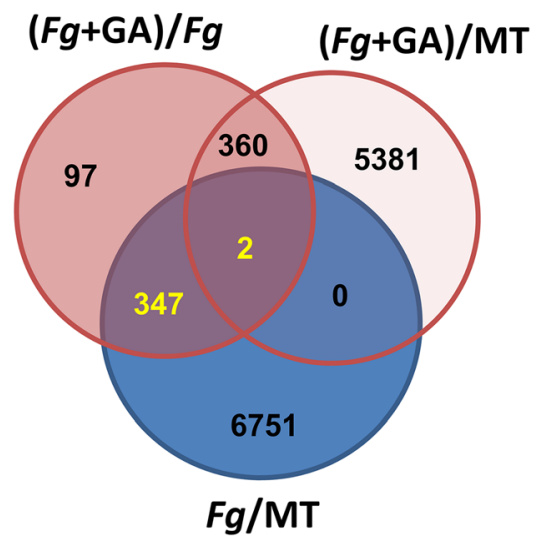

Fig. 4 Comparison of gene regulation elicited by GA in the presence of $F g$, with DEGs calculated compared to mock treated (MT) as well as Fg. In all cases, up-regulated genes are highlighted in shades of red, and down-regulated genes are highlighted in shades of blue. DEGs called in text are yellow. a Distribution of all up-regulated genes by $F g+G A$ as compared to $F g$, or Fg $+G A$ and Fg-alone compared to MT. b Distribution of all down-regulated genes by $F g+G A$ as compared to $F g$, or $F g+G A$ and $F g$-alone compared to MT. c Distribution of up-regulated genes by Fg-alone, but down-regulated by Fg + GA as they compared to Fg or to MT. d Distribution of all down-regulated genes by Fg-alone, but upregulated by $\mathrm{Fg}+\mathrm{GA}$ as they compared to $\mathrm{Fg}$ or to MT.

Wheat host phytohormone biosynthesis and signaling gene expression is altered by $\mathrm{Fg}$ challenge and phytohormone application

Targeted analysis of transcriptomic changes to 'Fielder' phytohormone biosynthetic and signaling pathways elicited by $\mathrm{Fg}$ challenge and phytohormone application were performed based on local sequence alignment (BlastP) of known homologs against the wheat DEGs. Wheat challenged with $\mathrm{Fg}$ alone elicited DEGs in classic phytohormone defense responses including up-regulating SA $(P A L)$, JA $(O P R 3)$ and ET $(A C S)$ biosynthetic genes. In terms of signaling, SA $(N P R 1)$ and a repressor of JA $(J A Z)$ were down-regulated, while JA (OCRA3) and ET (ETR1) signaling genes were up-regulated (Additional file 5: Figure S11, Additional file 1 Tabs 'S2' \& 'S3'). Fg-challenge also impacted wheat biosynthetic and signaling pathways of non-defense phytohormones
(Fig. 6) with some mixed modulation of key contributors to the mevalonate and terpenoid biosynthesis pathways that feed into ABA, GA, CK and BR biosynthetic pathways. Finally, $F g$ challenge down-regulated ABA biosynthetic genes $(\beta-C R T Z, A O X$, and $A 8 H)$, while upregulating two GA biosynthetic genes (GA20ox and GA2ox) and down-regulating a GA signaling repressor (DELLA; Additional file 5: Figures S12 \& S13).

The co-applied condition of $F g+\mathrm{ABA}$ elicited an upregulation of wheat DEGs in the ABA (HMGCR, ratelimiting NCED, $A 8 H)$, GA (GA2ox and GA20ox), ET $(A C O)$ and JA (OPR3) biosynthetic pathways (Additional file 5: Figures S12 \& S13). This induction of GA20ox and GA2ox wheat genes was consistent with the observed change in GA metabolites when comparing $\mathrm{Fg}$ challenged and $\mathrm{Fg}+\mathrm{ABA}$ treated wheat cultivar 'Fielder' phytohormone profiles (Table 4). The $F g+$ ABA 


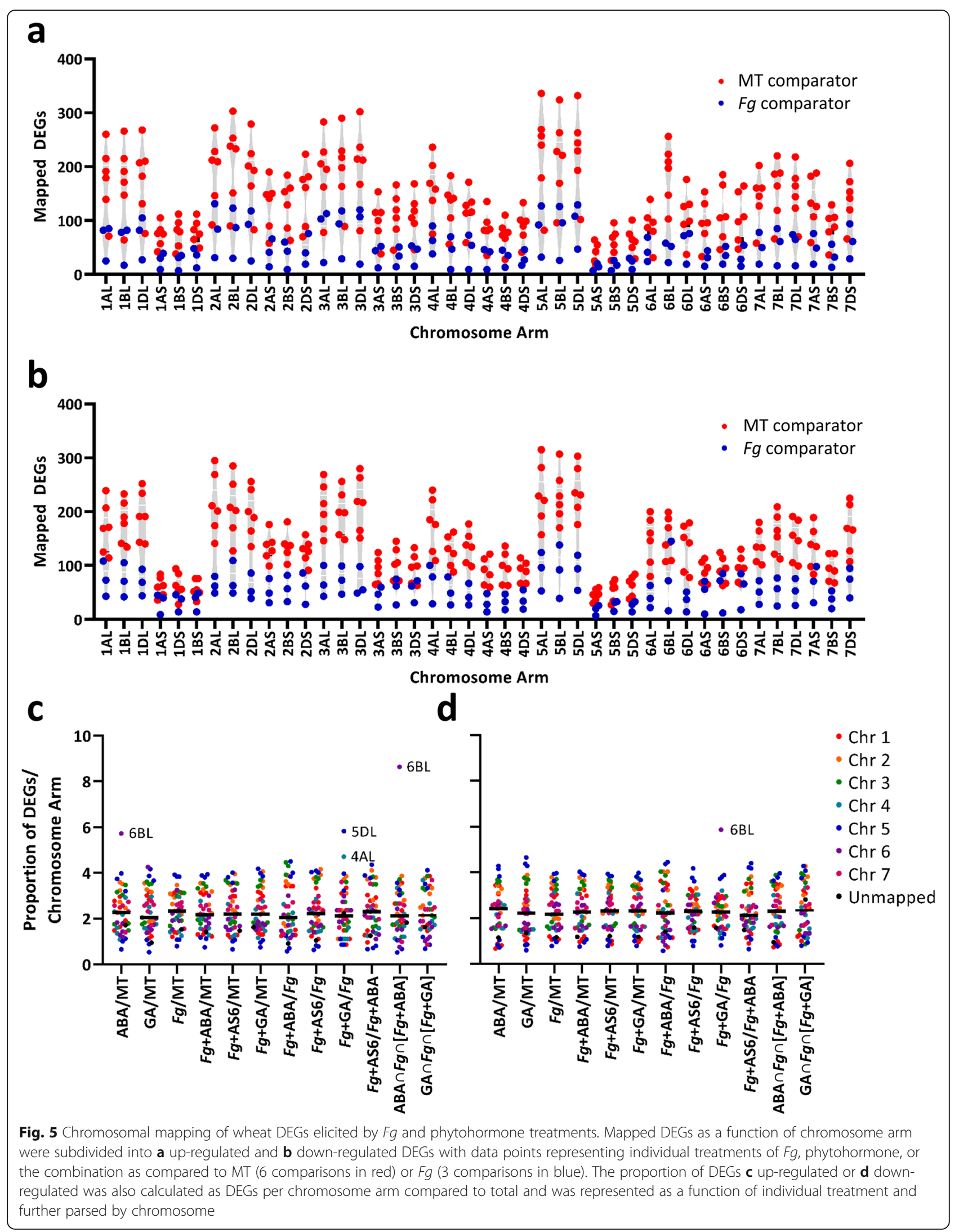




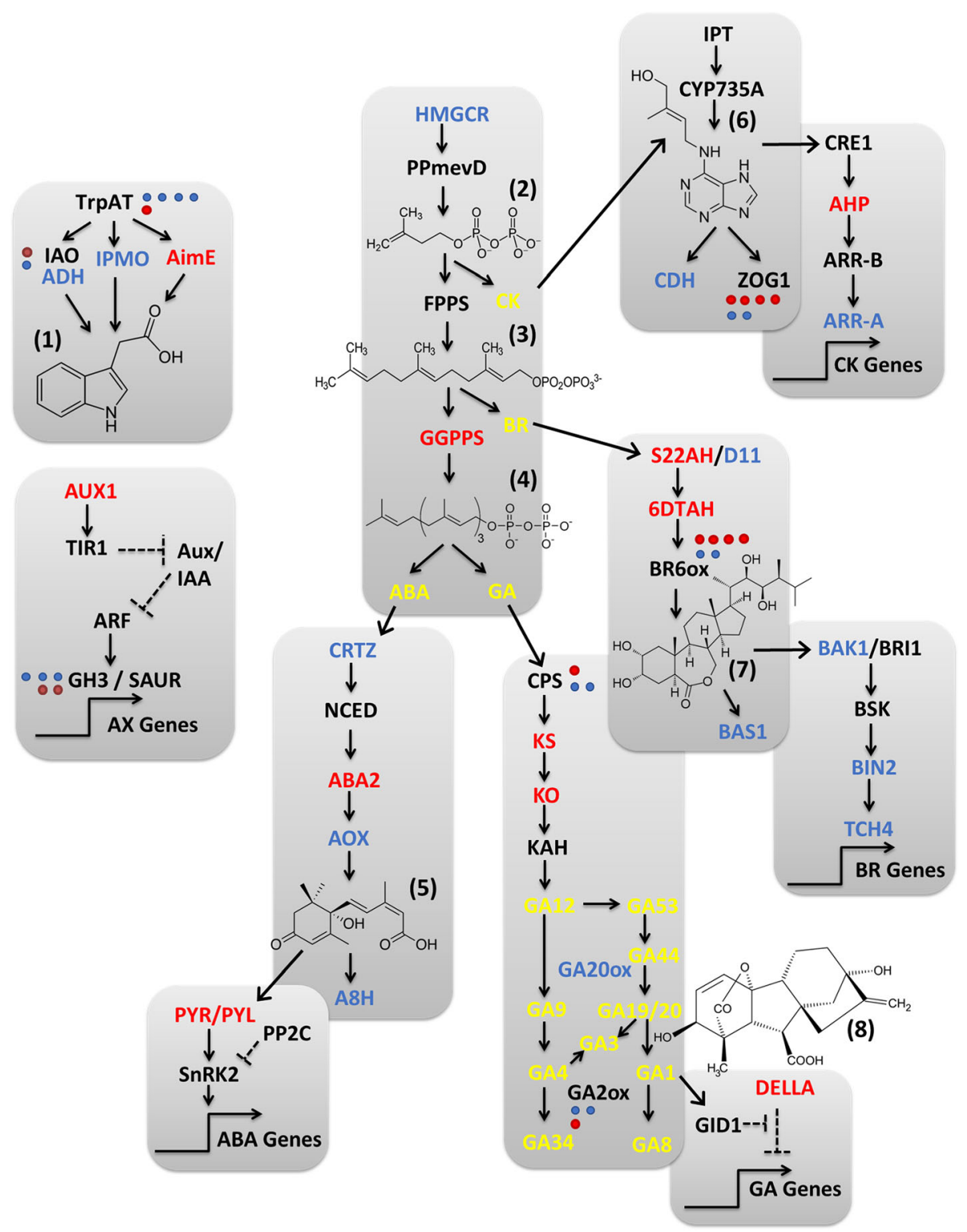

Fig. 6 Effect of $\mathrm{Fg}$ infection on expression of other (non-classical defense) 'Fielder' phytohormone biosynthesis and signaling pathway genes. Chemical structures are shown for (1) indole acetic acid, (2) isopentenyl diphosphate (3) farnesyl diphosphate (4) geranylgeranyl diphosphate (5) abscisic acid (6) transzeatin (7) brassinolide and (8) giberellin (GA3). Compound acronyms are highlighted in yellow. 'Fielder' gene acronyms are represented as up-regulated (red; $\log _{2} \mathrm{FC}>1.5$ ) and down-regulated (blue; $\log _{2} \mathrm{FC}<-1.5$ ), respectively (adj $p<0.01$ ) compared to mock treatment. Annotations were based on BlastP analysis selecting for transcripts with $>50 \%$ sequence identity to known phytohormone signaling pathway members as annotated in the KEGG database. See Additional file 1, Tabs S2 and S3 for detailed expression data

condition also yielded significant modulation of ABA signaling genes, including both suppression of multiple receptors and enhanced expression of multiple negative regulator PP2Cs, and up-regulation of SA (PR1), ET (ETR1), and BR (TCH4) signaling gene expression. These DEGs would be expected to reduce ABA signaling via two mechanisms and stimulate SA and ET signaling, with the latter two phytohormones both thought to have time-sensitive involvement in FHB defense $[3,6,8,9$,
12]. Meanwhile, the co-applied $F g+\mathrm{GA}$ suppressed the expression of GA biosynthetic genes $(K O$ and $K A H)$ and the negative regular DELLA involved in GA signaling. This condition also suppressed expression of ET (ETR1) and CK signaling genes ( $A-A R R$ and $A H P)$ and BR biosynthetic genes ( $S 22 A H$ and $B R 6 o x)$. The absence of an impact on wheat $A B A$ signaling gene expression by the $\mathrm{Fg}+\mathrm{GA}$ condition, and conversely GA signaling by the $\mathrm{Fg}+\mathrm{ABA}$ condition, is generally consistent with the 
a

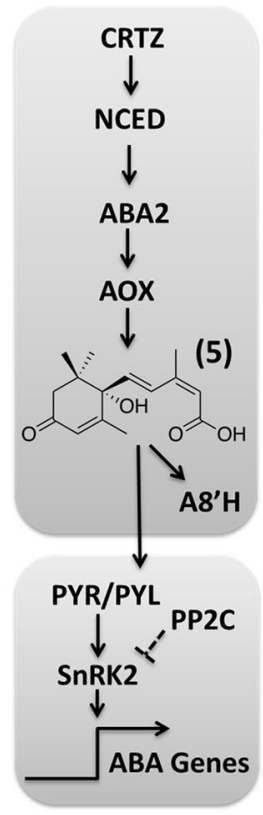

b
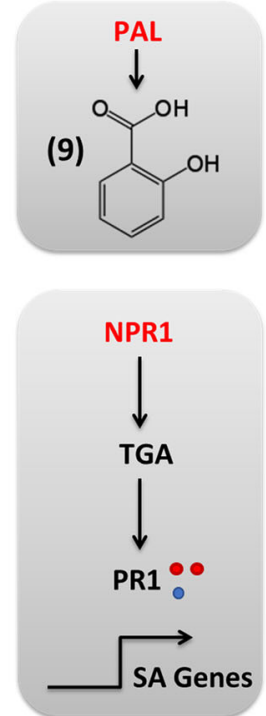
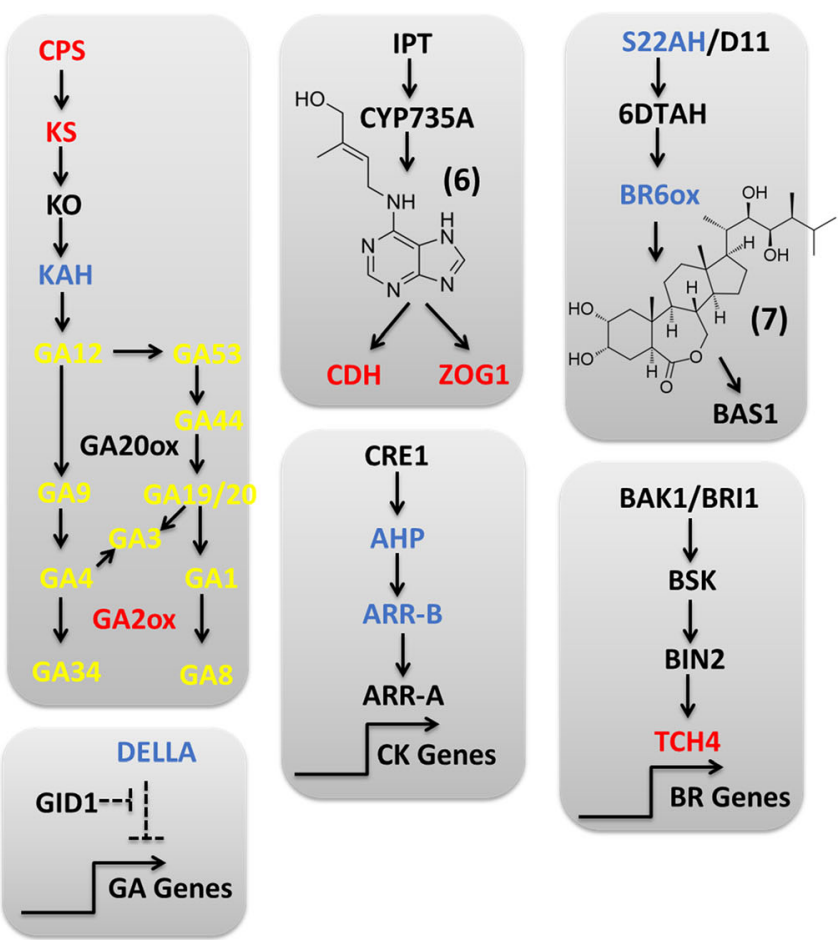
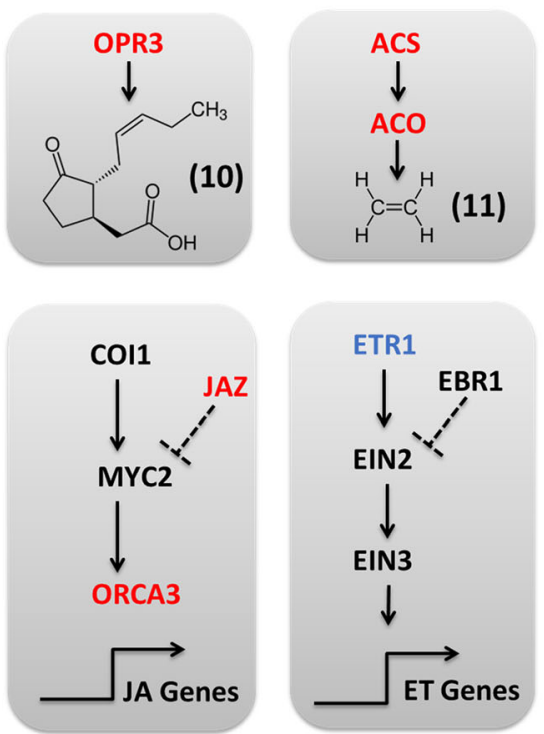

Fig. 7 Phytohormone biosynthetic and signaling responses arising from co-application of AS6 at the time of Fg infection, with DEGs calculated compared to $\mathrm{Fg}$ alone. a ABA and other affected (non-classical defense) hormone pathways with chemical structures for (5) abscisic acid, (6) trans-zeatin and (7) brassinolide. GA metabolite acronyms are highlighted in yellow. b Affected classical defense pathways, with chemical structure for (9) salicylic acid (10) jasmonic acid and (11) ethylene. Legend details are otherwise the same as in Fig. 6. See Additional file 1, Tabs S2 and S3 for details

above noted suppression of FUS3, an important regulator at the intersection of ABA and GA crosstalk [27], under all conditions with $\mathrm{Fg}$ challenge (Additional file 5: Figure S1). Finally, the co-applied condition of $F g$ AS6 up-regulated wheat DEGs in SA (PAL), JA (OPR3), ET
(ACS and ACO), GA (CPS, KS, and GA2ox), and CK $(C D H$ and ZOG1) biosynthesis while repressing genes in BR biosynthesis ( $S 22 A H$ and BR6ox). This condition also impacted wheat signaling gene expression with induction of SA (NPR1), JA (repressive JAZ and ORCA3), and 
Table $4 \mathrm{ABA}$ and GA phytohormones and their metabolites detected in 'Fielder' spikes upon Fg challenge. Phytohormones and their associated metabolite levels were detected in 'Fielder' spikes (normalized to dry weight (DW)) inoculated with $F g$ in the absence $(F g)$ or presence of either $\mathrm{ABA}(\mathrm{Fg}+\mathrm{ABA})$ or $\mathrm{GA}(F g+$ $G A)$. The ABA metabolites phaseic acid and dihydrophaseic acid are abbreviated PA and DPA, respectively, while undetected metabolites are denoted 'ND'. Phytohormone changes were evaluated with one-way ANOVA with Dunnett post-hoc comparisons (* $p \leq 0.05,{ }^{* *} p \leq 0.01,{ }^{* * *} p \leq 0.001,{ }^{* * * *} p \leq 0.0001$ )

\begin{tabular}{llll}
\hline $\begin{array}{l}\text { Metabolite } \\
\text { (ng/g DW) }\end{array}$ & Treatment & & \\
\cline { 2 - 4 } & $\boldsymbol{F g}$ & $\boldsymbol{F g}+\boldsymbol{A B A}$ & $\boldsymbol{F g}+\mathbf{G A}$ \\
\hline ABA & $143 \pm 37.4$ & $32,800 \pm 5500^{* * * *}$ & $75.1 \pm 21.6$ \\
DPA & $205 \pm 274$ & $49,400 \pm 10,600 * * *$ & $58.4 \pm 39.8$ \\
PA & $91.0 \pm 88.8$ & $55,500 \pm 18,100^{* *}$ & $34.6 \pm 17.9$ \\
7'-OH ABA & $155 \pm 51.1$ & $7900 \pm 2070^{* * *}$ & $77.6 \pm 21.9$ \\
neo-PA & $23.0 \pm 8.4$ & $975 \pm 216^{* * *}$ & $4.9 \pm 1.2$ \\
trans-ABA & $42.0 \pm 34.4$ & $1460 \pm 769 *$ & $7.6 \pm 2.4$ \\
GA1 & ND & $6.7 \pm 1.5$ & $249 \pm 22.3$ \\
GA3 & $37.0 \pm 16.7$ & $24.8 \pm 13.2$ & $343 \pm 13.4 * *$ \\
GA8 & $15.0 \pm 5.7$ & $11.2 \pm 2.3$ & $48.4 \pm 9.8 *$ \\
GA19 & $19.0 \pm 7.2$ & $19.0 \pm 2.5$ & $25.2 \pm 6.0$ \\
GA20 & ND & ND & $6.3 \pm 0.7$ \\
GA44 & ND & $10.0 \pm 0.6$ & $5.1 \pm 0.5$ \\
\hline
\end{tabular}

BR (TCH4) while repressing ET (ETR1), GA (the repressive DELLA), and CK (CDH and ZOG1) genes alone (Fig. 7). Interestingly, $F g+$ AS6 did not elicit changes to ABA metabolic or signaling pathways nor antagonistic changes to phytohormone pathways when compared to $F g+\mathrm{ABA}$ (except for DEGs corresponding to ACS in the ET pathway).

\section{Generation of a consensus model of wheat transcriptomic responses to $\mathrm{Fg}$ challenge}

In this work, the comparative transcriptomic responses of 'Fielder' are characterized; however, it remains unclear what proportion of, or trends in, this high-quality 'Fielder' transcriptome data have the greatest biological implications, especially considering disagreements between previous reports [6]. The results identified herein were compared with the four other FHB susceptible genotypes 'Roblin' [10, 11, 28], 'Shaw' [8], 'Stettler' and 'Muchmore' [9] to identify the strongest, consistent responses that may contribute to a consensus model of FHB infection. Findings are discussed if three or more genotypes reported significant expression changes in spikelet tissue. First, of the approximately 2600 'Fielder' key hub genes identified upon $\mathrm{Fg}$ challenge here (Additional file 2 Tab 'WheatDEGs'@Col-P), many were highly regulated by all genotypes. These changes include down-regulation of fatty acid hydroxylase superfamily (13 DEGs with mixed regulation of 5 additional DEGs), photosystem II $22 \mathrm{kDa}$ (3), PISTILLATA-like MADSbox transcription factor (2) and D-glycerate dehydrogenase/ hydroxypyruvate reductase and up-regulation of $\mathrm{C} 2$ domain-containing protein-like (5 DEGS with mixed regulation of 4 additional DEGs) and yellow stripe-like transporter 12 (4) (Additional file 8 Tab 'Hub-Genes'). Secondly, among the genes involved in the phytohormone biosynthetic pathways (Additional file 1, Tab S2), $\mathrm{Fg}$ challenge up-regulated ET (three ACS synthase genes), JA (OPR3), and SA (PAL) biosynthetic DEGs while also up-regulating JA (ORCA3) and auxin $(\mathrm{CH} 3)$ signaling DEGs (Additional file 1, Tab S3). Pathogen treatment consistently repressed auxin biosynthesis (IPMO and $\mathrm{ADH})$. There is also moderate agreement within the other comparative reports describing the upregulation of jasmonate ZIM-domain (7) and downregulation of pectinesterase (4) (Additional file 8, Tab Hub-genes). Third, an overlapping set of DEGs involved in aromatic and nitrogen metabolism are downregulated and include a CTP synthase, DNA-directed RNA polymerase, and phenylalanine ammonium lyase along with mixed changes among several other DEGs (Additional file $8 \mathrm{Tab}$ 'aromatic' and 'Cell N'). Thirteen additional up-regulated phenylalanine ammonium-lyase DEGs involved in cinnamic acid biosynthesis demonstrate substantial agreement between genotypes (Additional file 8 Tab 'cinnamic'). Fourth, a consensus pattern emerged herein with GA treatment downregulating and $F g$ up-regulating sets of DEGs. These DEGs were involved in heme (hemoglobin and cytochrome P450), oxidoreductase (cytochrome P450, polyphenyl oxidase, and aldehyde oxidase), and hydrolase $(\alpha /$ $\beta$ hydrolase superfamily) activities (Additional file 8 Tabs 'Heme', 'oxidoreductase', and 'hydrolase'). Finally, there is moderate agreement in the other four comparative genotypes reporting up-regulation of hydrolase ( $\beta$-amalase, $\beta$-fructofuranosidase, $\beta$-glucosidase, pectin acetylesterase, pectinesterase, and ribonuclease) and oxidoreductase (protochlorophyllide reductase, laccase, and respiratory burst oxidase) related DEGs (Additional file 8 Tabs 'hydrolase' and 'oxidoreductase').

\section{Discussion}

Comparative transcriptomic studies continue to be insightful in understanding general mechanisms modulating host resistance and susceptibility when challenged with $\mathrm{Fg}$ (reviewed in [6]). An encouraging flurry of recent publications emphasize roles for non-classical defense phytohormones in both resistance and susceptibility [7-11], suggesting new focused alternative mechanisms underlying the host-pathogen interaction. To 
date, these studies have highlighted variable responses between examined cultivars, an aspect which is further complicated by recent evidence suggesting that nonclassical defense phytohormones are also biosynthesized by $F g$ itself $[14,16,18,19]$. In this light, the expression of a putative fungal ABA biosynthetic cytochrome P450 homolog under all conditions containing $\mathrm{Fg}$ examined herein is noted (Additional file 5: Figure S2B), lending further support to the role for fungal-derived $A B A$ as an effector of infection, as has been proposed previously $[14,29]$.

\section{Assimilating 'Fielder' transcriptomic changes by comparing patterns of explicit features}

The wheat whole-transcriptome analyses reported here were aimed at characterizing treatment-elicited changes in 'Fielder' and $F g$ gene expression by distilling the tens of thousands of observations into distinct strings of DEG permutations using DEFE. These combinations of explicit characteristics may then be used to understand the relationship between gene regulation and phenotype, especially when considering multiple treatment types [22]. DEFE descriptors quantifying the veritable impact of $F g$ challenge on wheat gene expression, highlighted abundant 'Fielder' expression changes elicited by ABA or GA application alone, and described the unexpected DEG overlaps of $F g+$ AS6 with $F g+\mathrm{GA}(86 \%)$ and $F g+\mathrm{ABA}$ (23\%). Furthermore, by comparing individual DEFE descriptors from single phytohormone treatments with the intersection of several treatments (e.g., $\mathrm{ABA} F \mathrm{Fg} \cap[\mathrm{Fg}+$ $\mathrm{ABA}]$ ), DEGs elicited by ABA or GA alone were shown to be common in the co-application samples in 91 and 83\% of DEGs, respectively. By combining DEFE with topology overlap and gene association network analyses, more than 9500 'Fielder' DEGs were characterized as $\mathrm{Fg}$ related, where the most highly enriched genes are putatively involved in stress, cell structural integrity, membrane/lipid homeostasis, or molecular transport. As expected in 'Fielder' spikelets, many of these enriched genes are putatively involved in flowering [30-32]. Finally, FUS3 was identified as one notable gene in the network, having greater than 2000 connections. The FUS3 ortholog in Arabidopsis thaliana (AT3G26790) is known to be involved in positive regulation of ABA biosynthesis and negative regulation of GA biosynthesis [27]. As FUS3 gene expression was strongly suppressed across all $F g$-challenged conditions, the presence of the pathogen likely dysregulates ABA and GA crosstalk and may decouple their often-oppositional biological phenotypes. In additional to biosynthesis, the disruption of both $\mathrm{ABA}$ and GA signaling has been observed in the presence of Fusarium virulence factors [33], suggesting there may be multiple mechanisms used by the pathogen to dysregulate ABA and GA related plant responses.
Wheat transcriptomic responses to Fg challenge include ubiquitous traditional defense responses and more nuanced changes in non-classical defense phytohormones

$F g$-challenge of wheat varieties elicits an incontestable shift of host gene expression with noted variations in the expression of phytohormone biosynthetic and signaling pathway genes [8-11, 34]. When investigating 'Fielder' in this work, $F g$ challenge up-regulated both SA and JA biosynthetic pathways, notably with markers of late SA signaling highlighted, consistent with previous reports $[8,34]$; while mixed directions in differential gene expression of ET biosynthesis are juxtaposed to induction of one ET receptor gene, together suggesting ET pathways are being 'primed' for response. Beyond these classical defenses, $F g$-challenge also elicited mixed expression changes of biosynthetic and signaling pathways of ABA, GA, IAA, BR, and CK where GA and IAA biosynthetic genes were up-regulated, GA and BR signaling genes were down-regulated, and $A B A$ signaling genes were up-regulated.

When investigating cereal-Fusarium interactions using transcriptomic approaches, a variety of phytohormone responses have been observed which often do not agree between reports [6]. Wang et al. [9] may be most comparable to this work; therefore, a direct comparison may be more appropriate than a field-wide discussion in which little consensus is noted. Both works characterize up-regulation of genes involved in SA biosynthesis and SA, JA, ET, and ABA signaling. However, here we report increased JA and mixed ET biosynthetic gene expression changes, while Wang et al. characterize susceptible lines down-regulating JA and up-regulating ET biosynthetic genes with a contrasting increase in JA phytohormone concentration. Based on the consensus model of early biotrophic SA followed by later stage necrotrophic JA/ ET responses $[6,8,9]$, these differences may suggest that 'Fielder' tissues were analyzed while still transitioning to the later JA (increased biosynthesis) / ET ('primed' biosynthesis) response, whereas Wang et al.'s 'Settler' and 'Muchmore' varieties were characterized at a later infection state. This hypothesis is consistent with the 'Fielder' transcriptome being derived from early-stage at $24 \mathrm{~h}$ post inoculation (hpi), challenged spikelets while the 'Settler' and 'Muchmore' transcriptomes were derived from three pooled whole spikes with singly challenged spikelets (4 days post inoculation).

\section{ABA induces wheat DEGs observed in Fg infected tissues and may promote disease severity by misregulating phytohormone defense response and cell wall fortification mechanisms}

Unlike the model of early SA and later JA/ET responses triggered in wheat challenged with $F g[6,8,9]$, the gene 
expression changes upon application of individual phytohormones to wheat has not been coalesced into a single model. Perhaps the most comprehensive study of the differential gene expression has been the work of Qi et al. [11] where wheat spikelets were treated with IAA, GA, ABA, ET, CK, SA or JA (methyl jasmonate) and resultant DEGs characterized by microarray. Surprisingly, there were many contradictory findings when comparing the findings of Qi et al. and the transcriptomic changes presented here, namely in the number of DEGs identified per treatment and the relative proportionality between up- and down-regulated DEGs within a given treatment. These differences may be in part due to comparison between pooled wheat spikes vs treated spikelets or the comparison between two different genotypes, 'Roblin' vs 'Fielder'. It may also be method-dependent where wheat microarray analysis is limited to 50,00060,000 genes [11,34]; and additional stringency criteria must be applied to properly control for complications such as cross-hybridization and limited dynamic assay range (as reviewed in [35]). Despite these differences, both studies report a significant overlap between DEGs elicited by $\mathrm{Fg}$ challenge and $\mathrm{ABA}$ application as well as the ABA-dependent regulation of polyphenolic compound-related gene expression. Herein, exogenously applied ABA alone yielded a general repression in 'Fielder' where $57 \%$ of up-regulated and $75 \%$ of downregulated genes were also elicited by $F g$ challenge. Most of the genes (>90\%) significantly modified by ABA alone and $\mathrm{Fg}$ alone were also significantly modified in the coapplied $F g+\mathrm{ABA}$ condition, highlighting the relevance of findings from studies of $\mathrm{ABA}$-alone with respect to understanding the interaction of $\mathrm{ABA}$ with $\mathrm{Fg}$ infection. However, ABA treatment outcomes are not entirely overlapping with those elicited by either $F g$ or $F g+\mathrm{ABA}$. One notable case is the ABA down-regulated but $F g$ upregulated polyphenolic compound-related genes. Phenylpropanoid metabolism is involved in the biosynthesis of lignins, cinnamic acids, and flavonoids that are in turn used for cell wall fortification and $F g$ defense [36-40]. FHB resistant wheat and durum varieties have been shown to have key anatomical differences, improved cell wall structure including the additional lignin deposition, and greater accumulation of the cinnamic acid metabolite $p$-coumaric acid in infected tissues as compared to susceptible varieties [37, 41-43]. Furthermore, some polyphenolic compounds have documented anti-fungal activities $[44,45]$. In addition to the $\mathrm{ABA}$ alone treatment, the co-applied $F g+\mathrm{ABA}$ treatment also highlighted altered physical defense responses and nonclassical defense phytohormone signaling, specifically suppressing IAA and enhancing BR signaling. IAA signaling suppression is consistent with heightened classical defense responses and has been detected in susceptible near isogenic lines of the 2DL QTL, compared to more resistant lines [46, 47]. Enhanced BR signaling included the expression of a cell wall re-modelling xyloglucan endotransferase gene (TCH4) that may contribute to FHB susceptibility through inappropriately regulated cell wall modification. Together, wheat transcriptomic changes elicited within ABA treatment groups suggests that this phytohormone promotes FHB disease severity (this work, $[14,15])$ by inducing wheat gene expression changes necessary for $F g$ infection including misregulating classical defense phytohormone signaling and physical defense mechanisms, such as plant cell wall fortification and polyphenolic metabolism.

\section{GA induces global metabolic shifts in both the wheat host and $\mathrm{Fg}$ pathogen}

Historically, both genetic and chemical approaches have been applied to modulate GA metabolism for the improvement of agronomic wheat characteristics [48, 49]. It remains to be determined whether targeting GA metabolism could be a reasonable strategy for controlling FHB, based on the observation that GA-regulated wheat anthesis and plant height are strongly correlative with infection and disease severity [1,50-52]. Based on the transcriptomic profiles of wheat treated with diverse phytohormones, it was determined that ABA and GA elicit the strongest transcriptional antagonism of any phytohormone pair, with approximately $40 \%$ of their DEG being antagonistic [11]. These findings are also in agreement with previous reports of $\mathrm{ABA}$ promoting and GA suppressing FHB disease severity $[14,15]$. Here, exogenously applied GA alone resulted in a relatively even mix of up-regulated and down-regulated 'Fielder' genes including nearly 500 DEGs that directly opposed those elicited by $F g$ alone. GO enrichment suggest that these oppositional effects included down-regulated secondary metabolism and defense response genes, coincident with up-regulated primary metabolic processes. These GA derived DEGs describe a broader metabolic switch with moderate overlap compared to the primary carbohydrate, TCA, and nitrogen metabolic changes elicited by DON treatment [53]. It is also interesting to note that $F g$ transcriptionally regulates its own metabolism when transitioning from a biotrophic to necrotrophic state during the first $48 \mathrm{~h}$ post infection [54]. In Buhrow et al., [15], the effect of $\mathrm{Fg}+\mathrm{GA}$ co-application on Fusarium gene expression was characterized with this phytohormone eliciting DEGs broadly within amino acid, carbohydrate, and lipid metabolism and specifically regulating five genes in inorganic nitrogen or amino acid nitrogen metabolism. This coupling of GA and nitrogen metabolism has been previously described in the related Fusarium moniliforme [55]. Therefore, these findings suggest that GA may limit FHB severity through global 
metabolic changes on both the wheat host and $\mathrm{Fg}$ pathogen rather than through a select few defense pathways.

The Fg + AS6 treatment functions independently of ABAsignaling to antagonize 'Fielder' gene expression elicited by $A B A \cap F g \cap[F g+A B A]$ treatments

Co-application of AS6, a rationally designed ABA analog with a well-characterized ability to disrupt ABA receptor interactions in dicots [20], was investigated for its ability to elicit antagonistic transcriptomic responses compared to ABA. Notably ABA receptor functionalities in the wheat variety 'Thatcher' have been characterized previously [56]. In Fielder, $F g+$ AS6 elicited robust antagonist responses compared to DEGs elicited by $\mathrm{ABA} \cap F g \cap[F g+$ ABA]. Specifically, this treatment mitigated the upregulation of $\gamma$-glutamyl phosphate reductase and eukaryotic peptide chain release factor subunit 1-1 genes elicited by $\mathrm{ABA} \cap F g \cap[F g+\mathrm{ABA}]$. The expression of $\gamma$-glutamyl phosphate reductase has been tied to abiotic stress response [57]; while eukaryotic peptide chain release factor subunit 1-1 has been tied to plant growth and development including appropriate cell-wall lignification [58]. Fg + AS6 also robustly up-regulated expression of a leucine-rich repeat receptor-like protein kinase (LRRKs) and nitrate transporter 1.1 that were downregulated by $\mathrm{ABA} \cap F g \cap[F g+\mathrm{ABA}]$ treatments. LRRKs have been linked to FHB resistance in durum [50]; while nitrate transporter 1.1 has been suggested to function as a bridge between phytohormone responses during pathogen stress (reviewed in [59, 60]). As all the robust $\mathrm{Fg}+\mathrm{AS} 6$ responses can be tied to plant stress responses, and in some cases even FHB and phytohormone pathways, AS6 may in fact promote FHB resistance when applied in an appropriate developmental and sustained manner.

\section{Agreement between transcriptomic responses derived from several genotypes support a consensus model of the wheat-Fg interaction}

Previous transcriptomics reports of $\mathrm{Fg}$-challenged wheat highlighted up-regulated basal defenses, antagonism of pathogen-mediated modulation of phytohormone pathways, and classical phytohormone defense signaling [6165] with an early biotrophic SA followed by later stage necrotrophic JA/ET responses [6, 8, 9]. To additively contribute to this consensus model, differential gene expression were compared among five FHB susceptible wheat genotypes. Generally ubiquitous up-regulation of C2 domain-containing protein-like, jasmonate ZIMdomain, yellow stripe-like transporter 12, phenylalanine ammonium-lyases (cinnamic-acid metabolism) and down-regulation of hydrolases that putatively cleave fatty acids, pectins, ribose, and sugar moiety substrates were observed. Interestingly, ABA treatment also regulates
DEGs involved in cell wall-modifying polyphenolic and cinnamic acid metabolism and a saccharide hydrolase/ transferase, xyloglucan endotransferase. Meanwhile, GA treatment responses are targeted to metabolic processes like those elicited by DON treatment (discussed previously).

\section{Conclusions}

A comparative transcriptomic approach was applied in this work to elucidate the mechanisms by which ABA and GA affect wheat 'Fielder' FHB severity. Gene expression differences reported herein emphasize the vast impact of pathogen challenge and specifically highlight genes involved in defense response, cell structural integrity, molecular transport, and membrane/lipid metabolism as $F g$-responsive. $F g$ challenge notably downregulated expression of a key regulator of $\mathrm{ABA}$ and $\mathrm{GA}$ crosstalk, FUS3, supporting independent mechanisms by which ABA promotes and GA reduces FHB disease severity. Transcriptomic profiles presented herein emphasized that ABA co-application promotes disease by eliciting responses common to those elicited by the pathogen, misregulating defense responses by further exacerbating gene expression, and altering expression of cell wall fortification mechanisms. Unexpectedly, AS6 did not transcriptionally antagonize ABA signaling or biosynthesis in 'Fielder' but opposed other ABA- and Fgelicited responses, highlighting potential applications of ABA antagonists in future FHB research and disease mitigation efforts. In contrast, GA co-application elicited transcriptomic responses with diverse metabolic implications for both the 'Fielder' host and Fg pathogen. Such metabolic reprogramming may result in a less susceptible host and/or less virulent pathogen, especially at a time co-incident with $\mathrm{Fg}^{\prime}$ s biotrophic to necrotrophic lifestyle transition, ultimately reducing disease severity. Finally, the biological implications of these transcriptome responses on the wheat- $F g$ interaction were evaluated by assessing common findings across five wheat genotypes, additively contributing to a limited consensus model of disease response and severity.

\section{Methods}

Chemicals, phytohormones and $\mathrm{Fg}$ inoculum preparation Gibberellin A3 was purchased from Sigma-Aldrich (St. Louis, MO). The National Research Council Hormone Profiling Facility provided (+)-ABA, while 3 ' -hexasulfanyl(+)-ABA was synthesized as described in Takeuchi et al., [20] and provided by Kenneth Nelson and Suzanne Abrams at the University of Saskatchewan. Phytohormone stocks were solubilized in deionized water as sodium salts by $1.0 \mathrm{~N} \mathrm{NaOH}$ titration and stored at $-20^{\circ} \mathrm{C}$ in amber vials. Working solutions were made in deionized water and $\mathrm{pH}$ was adjusted to $7.0 \pm 0.05$. Fg GZ3639 [66] was 
propagated on potato dextrose agar (PDA; Sigma-Aldrich) at $25^{\circ} \mathrm{C}$ for 5 days. To obtain spores, carboxymethylcellulose (CMC) liquid media (1.5\% CMC (Sigma), 0.1\% $\mathrm{NH}_{4} \mathrm{NO}_{3}, 0.1 \% \mathrm{KH}_{2} \mathrm{PO}_{4}, 0.05 \% \mathrm{MgSO}_{4} \cdot 7 \mathrm{H}_{2} \mathrm{O}$, and $0.1 \%$ yeast extract) was inoculated with a marginal $5 \mathrm{~mm}$ square PDA plug and grown for 5 days at $27^{\circ} \mathrm{C}$, shaking at 170 $\mathrm{rpm}$. Macroconidia were isolated by filtering through one layer of cheese cloth and $25 \mu \mathrm{m}$ Miracloth filter (EMD Millipore; Billerica, MA), washed three times with sterile water, and quantified using a haemocytometer and light microscopy.

Propagation of plants, pathogen-challenge $+/$ - co-applied phytohormones, and phytohormone profiling

T. aestivum variety 'Fielder' [67], obtained from Dr. Mark Jordan (Agriculture and Agri-Food Canada, Morden, MB, Canada), was grown in Sunshine ${ }^{\mathrm{R}}$ Mix 8 (Sungrow Horticulture, Agawam, MA) and maintained in climate-controlled chambers with a $16 \mathrm{~h}$ photoperiod, at $25^{\circ} \mathrm{C}$ followed by $8 \mathrm{~h}$ of dark at $16^{\circ} \mathrm{C}$ every day. Plants were watered as needed, fertilized biweekly with 20-20-20 (N-P-K) and trimmed regularly prior to infection with $F g$ to remove yellowing material or material with any signs of disease. At the two-leaf stage plants were treated with Intercept ${ }^{\mathrm{TM}}$ (Bayer Crop Science, Calgary, AB) as an aphid preventative, as previously described [15, 34]. During anthesis, florets from a central spikelet (destined for RNA sequencing) or entire spikes (destined for phytophormone profiling) were point inoculated with $10 \mu \mathrm{L}$ of $5.0 \times 10^{4} \mathrm{Fg} \mathrm{GZ3639} \mathrm{macroconidial} \mathrm{suspension} \mathrm{or}$ deionized water (mock). For phytohormone application or co-application, this inoculum contained 1.0 mM ABA, GA3, or AS6. To promote infection, wheat plants were transferred to climate-controlled chambers with misting to achieve $90 \%$ humidity for $24 \mathrm{~h}$.

Phytohormone content in individual 'Fielder' spikes was determined at $24 \mathrm{hpi}$. For each condition $(F g, F g+$ ABA, $F g+G A$ ), one spike on five different plants was treated. Individual spikes served as a single tissue sample ( 5 spikes $=5$ biological replicates with no tissue pooling). These individual spikes were flash frozen and ground in liquid nitrogen. Phytohormones were extracted and quantified by UPLC/ESI-MS/MS at the National Research Council of Canada in Saskatoon, Canada, as described in [68-72]. Phytohormone content differences were analyzed with one-way ANOVA with Dunnett post-hoc comparisons using GraphPad Prism 6 (GraphPad Software, Inc. La Jolla, CA).

\section{RNA sequencing, data processing, and differential expression analyses}

A total of seven 'Fielder' spikelet treatments ( $F g, \mathrm{MT}$, ABA, GA, $F g+$ ABA, $F g+$ GA. $F g+$ AS6) were analyzed at $24 \mathrm{hpi}$, each consisting of five biological replicate central spikelet tissues. Total RNA was extracted from these central spikelets (35 samples in total), purified, quantified, and sequenced as described previously [15]. In short, RNA was purified using the RNeasy Plant Mini Kit (Qiagen, Mississauga, ON) using an oncolumn DNaseI treatment (Qiagen). RNA quality was evaluated using NanoDrop ND-8000 (NanoDrop, Wilmington, DE) and agarose gel electrophoresis. RNA library construction was completed using $1.0 \mu \mathrm{g}$ total RNA and the TruSeq RNA Sample Prep Kit v2 (Illumina, SanDiego, CA). Library quality (RNA integrity number $8.9 \pm 0.38$, Supplemental S1) was confirmed using the 2100 Bioanalyzer (Agilent Technologies Inc. Santa Clara, CA) equipped with a DNA 1000 chip. Library concentrations were determined by quantitative polymerase chain reaction (qPCR) using the KAPA SYBR FAST ABI Prism qPCR Kit (Kapa Biosystems, Wilmington, MA) and the StepOnePlus Real-Time PCR System (Applied Biosystems, Foster City, CA). RNA samples were multiplexed at a sequencing depth of five libraries per lane. Equimolar concentrations of the libraries were pooled and a final concentration of $12 \mathrm{pM}$ was used for clustering in cBOT (Illumina) flowcell lanes. The samples were then sequenced $(2 \times 101$ cycles, paired end reads) on the HiSeq2500 (Illumina) using the TruSeq SBS Kit v3-HS 200 cycles Kit (Illumina). The raw RNA-seq reads (available at GEO GSE137895) were preprocessed by trimming the adaptor sequences, filtering low-quality reads (Phred Score $\leq 20$ [73]), and eliminating short reads (length $\leq$ $20 \mathrm{bps}$ ) using software package FASTX-toolkit (default settings) [74]. After filtering, barcode and adaptor removal, an average of 27 million paired reads per sample was retained for subsequent read mapping through the RNA-seq data processing procedures. The IWGSC RefSeq v1.0 complete reference genome and corresponding annotation v1.0 were used as reference for the analysis of wheat RNA-seq data [21]. Following the recommendation of IWGSC, the chromosome partitioned version (161010_Chinese_Spring_v1.0_pseudomolecules_parts.fasta) was used and the gff3 file was reformatted accordingly. Only the high confidence gene models were considered in the mapping process. The $\mathrm{Fg}$ reference genome (Fusarium graminearum str. $\mathrm{PH}-$ 1) was obtained from EnsemblFungi (http://fungi. ensembl.org/ Release 35). Wheat and Fg genomes and annotation data from both species were combined into a single reference [75]. This combined reference genome contains 124,935 gene models, 14,145 from $F g$ and 110,790 from wheat. The cleaned RNA-seq reads in each sample were mapped using STAR v2.5.3a (default settings) [76] to generate gene-level read counts. DESeq2 [77] was used for data normalization and 
subsequent differentially expressed gene (DEG) analysis for each pairwise comparison. Normalized read counts along with $\log _{2}$ fold change and $p$-value, and adjusted $p$-values based on Benjamini and Hochberg procedure [78], were provided for downstream data analysis. The bioinformatics $\mathrm{R}$ script is available at the Github repository (https://github.com/DT-NRC/RNA-SeqDataProcessingProcedure).

\section{Data reduction and partitioning}

The output data from DESeq2 [77] was reduced in size to a set of differentially expressed genes. We applied the criteria of absolute $\log _{2} \mathrm{FC} \geq 1$, adjusted $p \leq 0.01$, and one of the pair of compared samples must be significantly expressed (i.e $\geq 10$ reads). Six informative pairwise comparisons between each treatment and MT and three pairs between co-application of $F g$ with a phytohormone and $F g$ alone and a comparison between co-applications of $F g$ with ABA and with AS6 (Fig. 1c, Table 1) were performed. The differential expression feature extraction method (DEFE [22];) was applied to partition the DEGs into various feature patterns. We compiled the six treatments compared to MT as one series of "M" patterns: $\mathrm{M}\left(\frac{A B A}{M T}, \frac{G A}{M T}, \frac{F g}{M T}, \frac{F g+A B A}{M T}, \frac{F g+A S 6}{M T}, \frac{F g+G A}{M T}\right)$ (Additional files 2 \& 3 Tab "DEFE stats"). For example, in the pattern M201110, at each comparison, " 0 " denotes no change, "1" for up- and "2" for down-regulation; this example pattern means a gene is down-regulated by ABA alone, has no significant change with respect to GA, whether applied with or without $\mathrm{Fg}$, but is up-regulated by $\mathrm{Fg}$ alone, $F g+\mathrm{ABA}$ and $F g+$ AS6. Similarly, we compiled the three combinations of respective phytohormones with $F g$ infection as compared with $F g$ infection alone in a series "F" patterns: $\mathrm{F}\left(\frac{F g+A B A}{F g}, \frac{F g+A S 6}{F g}, \frac{F g+G A}{F g}\right)$ (Additional file 2 Tab "DEFE stats").

\section{Clustering, correlation, network, gene ontology enrichment, and orthology analyses}

These analyses were done following the same methods described in [8]. Briefly, detailed methods for clustering, correlation and network analyses were provided in WGCNA R package [23] with the same modification as described in [8]; gene ontology enrichment analysis was performed using GOAL [79]; and reciprocal best hit BlastP was used for orthology determination.

\section{Phytohormone pathway gene identification and analyses} BlastP analyses of representative biosynthetic and signaling query sequences were performed against the DEGs derived in this study based on the Kyoto Encyclopedia of Genes and Genomes (KEGG) Database (cut off $>50 \%$ amino acid sequence identity). This yielded significant DEGs $\left(\log _{2} \mathrm{FC}>1.5\right.$ or $<-1.5$; adj $p<0.01$; to highlight the more highly modified) covering the spectrum of plant phytohormone biosynthesis and signaling pathways (Additional file 1 Tabs 'S2' \& 'S3' respectively).

\section{Abbreviations}

ABA: Abscisic acid; BR: Brassinosteroid; CK: Cytokinin; DEFE: Differential expression feature extraction; DEG: Differential gene expression; Fg: Fusarium graminearum; FHB: Fusarium head blight; ET: Ethylene; GA: Gibberellic acid; IAA: Indole acetic acid (auxin); JA: Jasmonic acid; SA: Salicylic acid

\section{Supplementary Information}

The online version contains supplementary material available at https://doi. org/10.1186/s12864-021-08069-0.

\begin{abstract}
Additional file 1: supplementary tables. This file contains four tables: (S1) RNA-seq read mapping statistics, (S2) 'Fielder' phytohormone biosynthetic pathways are affected by co-application of hormones with $\mathrm{Fg}$ challenge, compared to hormone- or Fg-challenge alone, (S3) 'Fielder' phytohormone signalling pathways are affected by co-application of hormones with Fg challenge, compared to hormone- or Fg-challenge alone, and (S4) distribution of phytohormone pathway genes over the chromosome arms.
\end{abstract}

Additional file 2. Differentially Expressed Wheat genes. This file contains three worksheets: (1) a list of 26,001 wheat DEGs, their expression values, cluster membership, gene network property, differential expression ( $\log _{2}$ FC and adj $p$ values), DEFE pattern, and correlation analysis, etc.; (2) DEFE statistics, number of genes belong to each DEFE pattern, full lists of Tables 2 and 3; (3) correlation analysis of the 58 clusters.

Additional file 3. Differentially Expressed Genes of Fusarium graminearum. This file contains two worksheets: (1) a list of 4872 Fg DEGs, including their expression values, differential expressions $\left(\log _{2} \mathrm{FC}\right.$ and adj $p$ values), and DEFE pattern.

Additional file 4. Enrichment index of various genes with their membership in various groups including DEGs, FHB-related genes, top $1 \%$ gene Network, and Key hub genes.

Additional file 5. 13 Supplementary Figures.

Additional file 6. Three series of Gene Ontology analyses in 32 worksheets plus a summary note worksheet: (1) FD series: ten lists of wheat genes down-regulated by Fg; (2) FU series: 19 lists of wheat genes up-regulated by $\mathrm{Fg}$; (3) F series: three groups of $\mathrm{Fg}$ genes differentially expressed as affected by AS6 and others.

Additional file 7. Twenty-six up-regulated genes by $\mathrm{Fg}$ alone were suppressed by $\mathrm{Fg}+\mathrm{AS6}$.

Additional file 8. Comparisons across five wheat genotypes susceptible to FHB. This file contains seven worksheets: (1) network hub genes; (2) genes involved in the aromatic metabolism; (3) genes involved in the cellular nitrogen metabolism; (4) genes involved in the cinnamic acid biosynthesis; (5) genes involved in the heme activity; (6) genes involved in the oxidoreductase activity; and (7) genes involved in the hydrolase activity.

\section{Acknowledgements}

We are grateful to Dr. L. Irina Zaharia at the Hormone Profiling Service, National Research Council of Canada, Saskatoon, Canada, for data collection and methodological input. We thank Dr. Susan McCormick at the United State Department of Agriculture for providing the F. graminearum strain GZ3639, Kenneth Nelson and Suzanne Abrams at the University of Saskatchewan for providing AS6. This manuscript represents NRC Communication \# 58262.

\section{Authors' contributions}

LMB performed all experiments, as well as contributed to the design of experiments, data analysis, interpretation and writing of the manuscript. ZL and DC contributed to data processing and analysis. TS contributed to analysis of the fungal data. NAF contributed to methods development, data analysis and writing of the manuscript. YP contributed to data processing, analysis, 
interpretation and writing of the paper. MCL conceived of the idea and contributed to experimental design, data analysis and interpretation and writing of the manuscript. All authors read and approved the final manuscript.

\section{Funding}

This research was funded to M.C.L and Y.P. by the Canadian Wheat Improvement Program, National Research Council of Canada (grants \# 008237 and \#011649), that reviewed the grants and provided funding for salaries, operational costs and services as well as access to facilities, but otherwise did not contribute intellectually to conceiving of the idea, planning of experiments or interpreting the data.

\section{Availability of data and materials}

The datasets generated and/or analyzed during the current study are available in the GEO repository, GSE137895.

\section{Declarations}

Ethics approval and consent to participate

Not Applicable.

\section{Consent for publication}

Not Applicable.

\section{Competing interests}

The authors declare that they have no competing interests.

\section{Author details}

'National Research Council of Canada, Aquatic and Crop Resources Development Research Centre, 110 Gymnasium Place, Saskatoon, SK S7N OM8, Canada. ${ }^{2}$ National Research Council of Canada, Digital Technologies Research Centre, 1200 Montreal Road, Ottawa, ON K1A OR6, Canada. ${ }^{3}$ University of Ottawa, Department of Chemistry and Biomolecular Sciences, 150 Louis-Pasteur Pvt, Ottawa, ON K1N 6N5, Canada. ${ }^{4}$ Agriculture and Agri-food Canada, Lethbridge Research and Development Centre, 5403 1st Ave, Lethbridge, AB T1J 4B1, Canada. ${ }^{5}$ National Research Council of Canada, Aquatic and Crop Resources Development Research Centre, 100 Sussex Drive, Ottawa, ON K1A OR6, Canada.

\section{Received: 27 September 2020 Accepted: 10 October 2021} Published online: 06 November 2021

\section{References}

1. Bai G, Shaner G. Management and resistance in wheat and barley to Fusarium head blight. Annu Rev Phytopathol. 2004;42(1):135-61. https://doi. org/10.1146/annurev.phyto.42.040803.140340.

2. Parry MAJ, Reynolds M, Salvucci ME, Raines C, Andralojc PJ, Zhu X-G, et al. Raising yield potential of wheat. II. Increasing photosynthetic capacity and efficiency. J Exp Bot. 2011;62(2):453-67. https://doi.org/10.1093/jxb/erq304.

3. Foroud NA, Pordel R, Goyal RK, Ryabova D, Chatterton S, Kovalchuk I. Chemical activation of the ethylene signalling pathway promotes wheat resistance to Fusarium graminearum. Phytopathology. 2019;109(5):796-803. https://doi.org/10.1094/PHYTO-08-18-0286-R.

4. Buerstmayr H, Ban T, Anderson JA. QTL mapping and marker-assisted selection for fusarium head blight resistance in wheat: a review. Plant Breed. 2009;128(1):1-26. https://doi.org/10.1111/j.1439-0523.2008.01550.x.

5. Cuperlovic-Culf M, Loewen M, Rajagopalan N, Surendra A. Perspectives on the specific targeting of Fusarium graminearum for the development of alternative head blight treatment approaches. Plant Pathol. 2017:66(9):1391403. https://doi.org/10.1111/ppa.12726.

6. Kazan K, Gardiner DM. Transcriptomics of cereal-Fusarium graminearum interactions: what we have learned so far. Mol Plant Pathol. 2018;19(3):76478. https://doi.org/10.1111/mpp.12561.

7. Biselli C, Bagnaresi P, Faccioli P, Hu X, Balcerzak M, Mattera MG, et al. Comparative transcriptome profiles of near-isogenic hexaploid wheat lines differing for effective alleles at the 2DL FHB resistance QTL. Front Plant Sci. 2018;9:37. https://doi.org/10.3389/fpls.2018.00037.

8. Pan Y, Liu Z, Rocheleau H, Fauteux F, Wang Y, McCartney C, et al. Transcriptome dynamics associated with resistance and susceptibility against Fusarium head blight in four wheat genotypes. BMC Genomics. 2018;19(1):642. https://doi.org/10.1186/s12864-018-5012-3.
9. Wang L, Li Q, Liu Z, Surendra A, Pan Y, Li Y, et al. Integrated transcriptome and phytohormone profiling highlight the role of multiple phytohormone pathways in wheat resistance against fusarium head blight. PLoS One. 2018; 13(11):e0207036. https://doi.org/10.1371/journal.pone.0207036.

10. Brauer EK, Rocheleau H, Balcerzak M, Pan Y, Fauteux F, Liu Z, et al. Transcriptional and hormonal profiling of Fusarium graminearum-infected wheat reveals an association between auxin and susceptibility. Physiol Mol Plant P. 2019;108:33-9. https://doi.org/10.1016/j.pmpp.2019.04.006.

11. Qi PF, Jiang YF, Guo ZR, Chen Q, Ouellet T, Zong LJ, et al. Transcriptional reference map of phytohormone responses in wheat spikes. BMC Genomics. 2019;20(1):390. https://doi.org/10.1186/s12864-019-5726-X.

12. Chen $X$, Steed A, Travella S, Keller B, Nicholson P. Fusarium graminearum exploits ethylene signalling to colonize dicotyledonous and monocotyledonous plants. New Phytol. 2009;182(4):975-83. https://doi.org/1 0.1111/j.1469-8137.2009.02821.X.

13. Sun $Y$, Xiao J, Jia X, Ke P, He L, Cao A, et al. The role of wheat jasmonic acid and ethylene pathways in response to Fusarium graminearum infection. Plant Growth Regul. 2016;80(1):69-77. https://doi.org/10.1007/s10725-0160147-1.

14. Qi PF, Balcerzak M, Rocheleau H, Leung W, Wei Y-M, Zheng Y-L, et al. Jasmonic acid and abscisic acid play important roles in host-pathogen interaction between Fusarium graminearum and wheat during the early stages of Fusarium head blight. Physiol Mol Plant P. 2016;93:39-48. https:// doi.org/10.1016/j.pmpp.2015.12.004.

15. Buhrow LM, Cram D, Tulpan D, Foroud NA, Loewen MC. Exogenous abscisic acid and gibberellic acid elicit opposing effects on Fusarium graminearum infection in wheat. Phytopathology. 2016;106(9):986-96. https://doi.org/10.1 094/PHYTO-01-16-0033-R.

16. Bhattacharya A, Kourmpetli S, Ward DA, Thomas SG, Gong F, Powers SJ, et al. Characterization of the fungal gibberellin desaturase as a 2-oxoglutaratedependent dioxygenase and its utilization for enhancing plant growth. Plant Physiol. 2012;160(2):837-45. https://doi.org/10.1104/pp.112.201756.

17. Luo K, Rocheleau H, Qi P-F, Zheng Y-L, Zhao H-Y, Ouellet T. Indole-3-acetic acid in Fusarium graminearum: identification of biosynthetic pathways and characterization of physiological effects. Fungal Biol. 2016;120(9):1135-45. https://doi.org/10.1016/j.funbio.2016.06.002.

18. Vrabka J, Niehaus E-M, Münsterkötter M, Proctor RH, Brown DW, Novák O, et al. Production and role of hormones during interaction of Fusarium species with maize (Zea mays L.) seedlings. Front Plant Sci. 2019;9:1936z.

19. Svoboda T, Parich A, Güldener U, Schöfbeck D, Twaruschek K, Václavíková $\mathrm{M}$, et al. Biochemical characterization of the Fusarium graminearum candidate ACC-deaminases and virulence testing of knockout mutant strains. Front Plant Sci. 2019;10:1072.

20. Takeuchi J, Okamoto M, Akiyama T, Muto T, Yajima S, Sue M, et al. Designed abscisic acid analogs as antagonists of PYL-PP2C receptor interactions. Nat Chem Biol. 2014;10(6):477-82. https://doi.org/10.1038/nchembio.1524.

21. IWGSC. Shifting the limits in wheat research and breeding using a fully annotated reference genome. Science. 2018;361:eaar7191.

22. Pan Y, Li Y, Liu Z, Surendra A, Wang L, Foroud NA, et al. Differential expression feature extraction (DEFE) and its application in RNA-seq data analysis. bioRxiv. 2019. https://doi.org/10.1101/511188.

23. Langfelder $\mathrm{P}$, Horvath S. WGCNA: an R package for weighted correlation network analysis. BMC Bioinformatics. 2008;9(1):559. https://doi.org/10.11 86/1471-2105-9-559.

24. Ritchie $\mathrm{S}$, Gilroy $\mathrm{S}$. Abscisic acid signal transduction in the barley aleurone is mediated by phospholipase D activity. Proc Natl Acad Sci U S A. 1998;95(5): 2697-702. https://doi.org/10.1073/pnas.95.5.2697.

25. Chono M, Matsunaka H, Seki M, Fujita M, Kiribuchi-Otobe C, Oda S, et al. Isolation of a wheat (Triticum aestivum L.) mutant in ABA 8'-hydroxylase gene: effect of reduced ABA catabolism on germination inhibition under field condition. Breed Sci. 2013;63:104-15.

26. Prada D, Romagosa I, Ullrich SE, Molina-Cano JL. A centromeric region on chromosome 6(6H) affects dormancy in an induced mutant in barley. J Exp Bot. 2005;56(409):47-54. https://doi.org/10.1093/jxb/erio05.

27. Tsai AY, Gazzarrini S. AKIN10 and FUSCA3 interact to control lateral organ development and phase transitions in Arabidopsis. Plant J. 2012;69(5):80921. https://doi.org/10.1111/j.1365-313X.2011.04832.x.

28. Pan Y, Ouellet T, Phan S, Tchagang A, Fauteux F, Tulpan D. Digitization of trait representation in microarray data analysis of wheat infected by Fusarium graminearum. Proceedings of the 2015 IEEE Conference on 
Computational Intelligence in Bioinformatics and Computational Biology. 2015. August 12-15, Niagara Falls, Canada.

29. Takino J, Kozaki T, Sato Y, Liu C, Ozaki T, Minami A, et al. Unveiling biosynthesis of the phytohormone abscisic acid in fungi: unprecedented mechanism of core scaffold formation catalyzed by an unusual sesquiterpene synthase. J Am Chem Soc. 2018;140(39):2392-12395. https://doi.org/10.1021/jacs.8b08925.

30. Becker A, Theissen G. The major clades of MADS-box genes and their role in the development and evolution of flowering plants. Mol Phylogenet Evol. 2003;29(3):464-89. https://doi.org/10.1016/S1055-7903(03)00207-0.

31. Cheung AY, Niroomand S, Zou YJ, Wu HM. A transmembrane formin nucleated subapical actin assembly and controls tip-focused growth in pollen tubes. Proc Natl Acad Sci U S A. 2010;107(37):16390-5. https//doi.org/10.1073/pnas.1008527107.

32. Bosch M, Helper PK. Pectin methylesterases and pectin dynamics in pollen tubes. Plant Cell. 2005;17(12):3219-26. https:/doi.org/10.1105/tpc.105.037473.

33. Kazan K, Lyons R. Intervention of phytohormone pathways by pathogen effectors. Plant Cell. 2014;26(6):2285-309. https://doi.org/10.1105/tpc.114.125419.

34. Foroud NA, Ouellet $\mathrm{T}$, Laroche A, Oosterveen B, Jordan MC, Ellis BE, et al. Differential transcriptome analyses of three wheat genotypes reveal different host response pathways associated with fusarium head blight and trichothecene resistance. Plant Pathol. 2012;61 (2):296-314. https://doi.org/1 0.1111/j.1365-3059.2011.02512.x.

35. Fathallah-Shaykh HM. Microarrays applications and pitfalls. Arch Neurol. 2005;62(11):1669-72. https://doi.org/10.1001/archneur.62.11.1669.

36. Gunnaiah R, Kushalappa AC, Duggavathi R, Fox S, Somers DJ. Integrated metabolo-proteomic approach to decipher the mechanisms by which wheat QTL (Fhb1) contributes to resistance against Fusarium graminearum. PLoS One. 2012;7(7):e40695. https://doi.org/10.1371/journal.pone.0040695.

37. Lionetti V, Giancaspro A, Fabri E, Giove SL, Reem N, Zabotina OA, et al. Cell wall traits as potential resources to improve resistance of durum wheat against Fusarium graminearum. BMC Plant Biol. 2015;15(1):6. https://doi. org/10.1186/s12870-014-0369-1.

38. Dhokane D, Karre S, Kushalappa AC, McCartney C. Integrated metabolotranscriptomics reveals Fusarium head blight candidate resistance genes in wheat QTL-Fhb2. PLoS One. 2016;11(5):e0155851. https://doi.org/10.1371/journal.pone.01 55851.

39. Kage U, Karre S, Kushalappa AC, McCartney C. Identification and characterization of a Fusarium head blight resistance gene TaACT in wheat QTL-2DL. Plant Biotechnol J. 2017;15(4):447-57. https://doi.org/10.1111/pbi.12641.

40. Karre S, Kumar A, Yogendra K, Kage U, Kushalappa A, Charron JB. HvWRKY23 regulates flavonoid glycoside and hydroxycinnamic acid amide biosynthetic genes in barley to combat Fusarium head blight. Plant Mol Biol. 2019;100(6): 591-605. https://doi.org/10.1007/s11103-019-00882-2.

41. Kang Z, Buchenauer H. Ultrastructural and cytochemical studies on the infection of wheat spikes by Fusarium culmorum as well as on degradation of cell wall components and localization of mycotoxins in the host tissue. Mycotoxin Res. 2000;16(Suppl 1):1-5. https://doi.org/10.1007/BF02942968.

42. Mohammadi M, Kazemi H. Changes in peroxidase and polyphenol oxidase activities in susceptible and resistant wheat heads inoculated with Fusarium graminearum and induced resistance. Plant Sci. 2002;162(4):491-8. https:// doi.org/10.1016/S0168-9452(01)00538-6.

43. Siranidou E, Kang Z, Buchenauer H. Studies on symptom development, phenolic compounds and morphological defence responses in wheat cultivars differing in resistance to Fusarium head blight. J Phytopathol. 2003; 150(4-5):200-8. https://doi.org/10.1046/j.1439-0434.2002.00738.x.

44. Cuperlovic-Culf M, Rajagopalan N, Tulpan D, Loewen MC. Metabolomics and cheminformatics analysis of antifungal function of plant metabolites. Metabolites. 2016;6:E31.

45. Rajagopalan N, Lu Y, Burton IW, Monteil-Rivera F, Halasz A, Reimer E, et al. A phenylpropanoid diglyceride associates with the leaf rust resistance $L r 34$ res gene in wheat. Phytochemistry. 2020;178:112456. https:/doi.org/10.1016/j.phytochem.202 0.112456 .

46. Denance N, Sánchez-Vallet A, Goffner D, Molina A. Disease resistance or growth: the role of plant phytohormones in balancing immune responses and fitness costs. Front Plant Sci. 2013;4:155. https://doi.org/10.3389/fpls.2013.00155.

47. Long XY, Balcerzak M, Gulden S, Cao W, Fedak G, Wei Y-M, et al. Expression profiling identifies differentially expressed genes associated with the fusarium head blight resistance QTL 2DL from the wheat variety Wuhan-1. Physiol Mol Plant P. 2015;90:1-11. https://doi.org/10.1016/.pmpp.2015.02.002.

48. Peng J, Richards DE, Hartley NM, Murphy GP, Devos KM, Flintham JE, et al. Green revolution genes encode mutant gibberellin response modulators. Nature. 1999;400(6741):256-61. https://doi.org/10.1038/22307.
49. Yang W, Yu Z, Yu S, Fan G, Han H, Dong Z, et al. Effect of uniconazole waterless dressing see on yield of wheat. Acta Agron Sin. 2004:30:502-6.

50. Sari E, Cabral AL, Polley B, Tan Y, Hsueh E, Konkin DJ, et al. Weighted gene co-expression network analysis unveils gene networks associated with the Fusarium head blight resistance in tetraploid wheat. BMC Genomics. 2019; 20(1):925. https://doi.org/10.1186/s12864-019-6161-8.

51. Buerstmayr M, Lemmens M, Steiner B, Buerstmayr H. Advanced backcross QTL mapping of resistance to Fusarium head blight and plant morphological traits in a Triticum macha $\times$ T. aestivum population. Theor Appl Genet. 2011;123(2):293-306. https://doi.org/10.1007/s00122-011-1584-x.

52. Buerstmayr M, Huber K, Heckmann J, Steiner B, Nelson JC, Buerstmayr H. Mapping of QTL for Fusarium head blight resistance and morphological and developmental traits in three backcross populations derived from Triticum dicoccum $\times$ Triticum durum. Theor Appl Genet. 2012;125(8):1751-65. https://doi.org/10.1007/s00122-012-1951-2.

53. Warth B, Parich A, Bueschl C, Schoefbeck D, Neumann NKN, Kluger B, et al. GC-MS based targeted metabolic profiling identifies changes in the wheat metabolome following deoxynivalenol treatment. Metabolomics. 2015;11(3): 722-38. https://doi.org/10.1007/s11306-014-0731-1.

54. Boedi S, Berger H, Sieber C, Münsterkötter M, Maloku I, Warth B, et al. Comparison of Fusarium graminearum transcriptomes on living or dead wheat differentiates substrate-responsive and defense-responsive genes. Front Microbiol. 2016;7:1113.

55. Mihlan M, Homann V, Liu TW, Tudzynski B. AREA directly mediates nitrogen regulation of gibberellin biosynthesis in Gibberella fujikuroi, but its activity is not affected by NMR. Mol Microbiol. 2003;47(4):975-91. https//doi.org/10.1046/.1365-2 958.2003.03326x.

56. Gordon C, Rajagopalan N, Riseeuw E, Surpin M, Ball F, Barber C, et al. Identification of $T$ aestivum abscisic acid receptors and evidence of their role in Fusarium head blight. PLoS One. 2016;11:e0164996.

57. Wang L, He X, Guo J, Shen Y, Huang Z. The expression of wheat TaSTG gene can enhance salt tolerance in plants. Plant Biosyst. 2013;147(2):451-8. https://doi.org/10.1080/11263504.2013.769469.

58. Petsch KA, Mylne J, Botella JR. Cosuppression of eukaryotic release factor 11 in Arabidopsis affects cell elongation and radial cell division. Plant Physiol. 2005;139(1):115-26. https://doi.org/10.1104/pp.105.062695.

59. Fan X, Naz M, Fan X, Xuan W, Miller A, Xu G. Plant nitrate transporters: from gene function to application. J Exp Bot. 2017;68(10):2463-75. https:/doi.org/10.1093/jxb/ enX011.

60. Krouk G. Hormones and nitrate: a two-way connection. Plant Mol Biol. 2016; 91(6):599-606. https://doi.org/10.1007/s11103-016-0463-x.

61. Rawat N, Pumphrey MO, Liu S, Zhang X, Tiwari VK, Ando K, et al. Wheat Fhb1 encodes a chimeric lectin with agglutinin domains and a poreforming toxin-like domain conferring resistance to Fusarium head blight. Nat Genet. 2016;48(12):1576-80. https://doi.org/10.1038/ng.3706.

62. Kage U, Yogendra KN, Kushalappa AC. TaWRKY70 transcription factor in wheat QTL-2DL regulates downstream metabolite biosynthetic genes to resist Fusarium graminearum infection spread within spike. Sci Rep. 2017; 7(1):42596. https://doi.org/10.1038/srep42596.

63. Li G, Zhou J, Jia H, Gao Z, Fan M, Luo Y, et al. Mutation of a histidine-rich calcium-binding-protein gene in wheat confers resistance to Fusarium head blight. Nat Genet. 2019;51(7):1106-12. https://doi.org/10.1038/s41588-0190426-7.

64. Su Z, Bernardo A, Tian B, Chen H, Wang S, Ma H, et al. A deletion mutation in TaHRC confers Fhb1 resistance to Fusarium head blight in wheat. Nat Genet. 2019;51(7):1099-105. https://doi.org/10.1038/s41588-019-0425-8.

65. Fauteux F, Wang Y, Rocheleau H, Liu Z, Pan Y, Fedak G, et al. Characterization of QTL and eQTL controlling early Fusarium graminearum infection and deoxynivalenol levels in a Wuhan 1 x Nyubai doubled haploid wheat population. BMC Plant Biol. 2019;19(1):536. https://doi.org/10.1186/ s12870-019-2149-4.

66. Proctor RH, Hohn TM, McCormick SP. Reduced virulence of Gibberella zeae caused by disruption of a tricothecene toxin biosynthetic gene. Mol Plant Microbe In. 1995;8(4):593-601. https://doi.org/10.1094/MPMI-8-0593.

67. Sunderman DW, Bruinsma B. Registration of four wheat cultivars. Crop Sci. 1975;15(1):104-5. https://doi.org/10.2135/cropsci1975.0011183X00150001004 $8 \mathrm{x}$.

68. Abrams SR, Nelson K, Abrose SJ. Deuterated abscisic acid analogs for mass spectroscopy and metabolism studies. J Labelled Compd Rad. 2003;46(3): 273-83. https://doi.org/10.1002/jlcr.670. 
69. Lulsdorf MM, Yuan HY, Slater SMH, Vandenberg A, Han X, Zaharia LI, et al. Endogenous hormone profiles during early seed development of $C$. arietinum and C. anatolicum. J Plant Growth Regul. 2013;71(2):191-8. https:// doi.org/10.1007/s10725-013-9819-2.

70. Galka MM, Ambrose SJ, Ross ARS, Abrams SR. Synthesis of deuterated jasmonates for mass spectroscopy and metabolism studies. J Labelled Compd Rad. 2005;48(11):797-809. https://doi.org/10.1002/jlcr.994.

71. Ross ARS, Ambrose SR, Cutler AJ, Feurtado JA, Kermode AR, Nelson K, et al. Determination of endogenous and supplied deuterated abscisic acid in plant tissues by high performance liquid chromatography-electrospray ionization tandem mass spectroscopy with multiple reaction monitoring. Anal Biochem. 2004;329(2):324-33. https://doi.org/10.1016/j.ab.2004.02.026.

72. Zaharia LI, Galka MM, Ambrose SJ, Abrams SR. Preparation of deuterated abscisic acid metabolites for use in mass spectroscopy and feeding studies. J Labelled Compd Rad. 2005;48(6):435-45. https://doi.org/10.1002/jlcr.939.

73. Ewing B, Hillier L, Wendl MC, Green P. Base-calling of automated sequencer traces using phred. I. Accuracy assessment. Genome Res. 1998;8(3):175-85. https://doi.org/10.1101/gr.8.3.175.

74. Hannon GJ. FASTX-Toolkit. 2010. http://hannonlab.cshl.edu/fastx_toolkit.

75. Liu Z, Li Y, Pan Y, Wang L, Ouellet T, Fobert P. Strategy in wheat-Fusarium dual-genome RNA-seq data processing. bioRxiv. 2019. https://doi.org/10.11 01/2019.12.16.878124.

76. Dobin A, Davis CA, Schlesinger F, Drenkow J, Zaleski C, Jha S, et al. STAR: ultrafast universal RNA-seq aligner. Bioinformatics. 2013;29(1):15-21. https:// doi.org/10.1093/bioinformatics/bts635.

77. Love MI, Huber W, Anders S. Moderated estimation of fold change and dispersion for RNA-seq data with DESeq2. Genome Biol. 2014;15(12):550. https://doi.org/10.1186/s13059-014-0550-8

78. Benjamini $Y$, Hochberg Y. Controlling the false discovery rate: a practical and powerful approach to multiple testing. J R Stat Soc Ser B Methodol. 1995;57(1):289-300. https://doi.org/10.1111/j.2517-6161.1995.tb02031.x.

79. Tchagang AB, Gawronski A, Bérubé H, Phan S, Famili F, Pan Y. GOAL: a software tool for assessing biological significance of genes groups. BMC Bioinformatics. 2010;11(1):229. https://doi.org/10.1186/1471-2105-11-229.

\section{Publisher's Note}

Springer Nature remains neutral with regard to jurisdictional claims in published maps and institutional affiliations.

Ready to submit your research? Choose BMC and benefit from:

- fast, convenient online submission

- thorough peer review by experienced researchers in your field

- rapid publication on acceptance

- support for research data, including large and complex data types

- gold Open Access which fosters wider collaboration and increased citations

- maximum visibility for your research: over $100 \mathrm{M}$ website views per year

At $\mathrm{BMC}$, research is always in progress.

Learn more biomedcentral.com/submissions 